\title{
The Transcriptional Response of Hybrid Poplar (Populus trichocarpa x P. deltoides) to Infection by Melampsora medusae Leaf Rust Involves Induction of Flavonoid Pathway Genes Leading to the Accumulation of Proanthocyanidins
}

\author{
Manoela Miranda, ${ }^{1}$ Steven G. Ralph, ${ }^{2}$ Robin Mellway, ${ }^{1}$ Rick White, ${ }^{3}$ Michele C. Heath, ${ }^{1}$ Jörg BohImann, ${ }^{2,4}$ \\ and C. Peter Constabel ${ }^{1}$ \\ ${ }^{1}$ Centre for Forest Biology and Department of Biology, University of Victoria, Victoria, BC, Canada; ${ }^{2}$ Michael Smith \\ Laboratories, University of British Columbia, Vancouver, BC, Canada; ${ }^{3}$ Department of Statistics, University of British \\ Columbia, Vancouver, BC, Canada; ${ }^{4}$ Departments of Forest Sciences and Botany, University of British Columbia, \\ Vancouver, BC, Canada
}

Submitted 14 October 2006. Accepted 13 February 2007.

\begin{abstract}
The transcriptional response of hybrid poplar (Populus trichocarpa $\times P$. deltoides) to poplar leaf rust (Melampsora medusae) infection was studied using the Populus $15.5 \mathrm{~K}$ cDNA microarray. Pronounced changes in the transcriptome were observed, with approximately $20 \%$ of genes on the array showing either induction or repression of transcription within the 9-day infection timecourse. A small number of pathogen-defense genes encoding PR-1, chitinases, and other pathogenesis-related proteins were consistently upregulated throughout the experimental period, but most genes were affected only at individual timepoints. The largest number of changes in gene expression was observed late in the infection at 6 to 9 days postinoculation (dpi). At these timepoints, genes encoding enzymes required for proanthocyanidin (condensed tannin) synthesis were upregulated dramatically. Phytochemical analysis confirmed that, late in the infection, proanthocyanidin levels increased in infected leaves. Strongly M. medusae-repressed genes at 9 dpi included previously characterized wound- and herbivore-induced defense genes, which suggests antagonism between the tree responses to insect feeding and $M$. medusae infection. In this highly compatible plant-pathogen interaction, we postulate that the biotrophic pathogen evades detection and suppresses early host responses.
\end{abstract}

Additional keywords: condensed tannins, forest tent caterpillar, fungal pathogen, phenylpropanoid metabolism.

M. Miranda and S. G. Ralph contributed equally to this work as first authors. Corresponding author: C. Peter Constabel; E-mail: cpc@uvic.ca; Fax: +1205-721-6611.

Current address for M. Miranda: Plant Breeding and Genetics Section, International Atomic Energy Agency, Vienna, Austria.

Current address for S. G. Ralph: Department of Biology, University of North Dakota, Grand Forks, ND, U.S.A.

* The $\boldsymbol{e}$-Xtra logo stands for "electronic extra" and indicates the online version includes supplemental material not included in the print edition. Four additional tables are available online.
Poplar (Populus spp.) is an established model system for genomic studies in angiosperm tree biology (Tuskan et al. 2006). The development and application of genomics tools to the biology of Populus has been a key development in the quest to understand the evolution, function, and adaptation of the genome of a long-lived, perennial, woody plant (Brunner et al. 2004; Strauss and Martin 2004; Tuskan et al. 2004, 2006). Genomic resources for Populus include large expressed sequence tag (EST) and full-length cDNA collections, DNA microarrays, high-density genetic maps, application of TILLING (targeting induced local lesions in genomes) as a single nucleotide polymorphism discovery tool, and the availability of a physical map and genome sequence of $P$. trichocarpa (Andersson et al. 2004; Gilchrist et al. 2006; Ralph et al. 2006; Sterky et al. 2004; Tuskan et al. 2006; Yin et al. 2004). These tools build on the other advantages of poplar as an experimental system, in particular its rapid growth from vegetative cuttings, a relatively short generation time (compared with many other tree species), and susceptibility to Agrobacterium-mediated transformation (Busov et al. 2005; Han et al. 2000). Recently, poplar has also received much renewed attention as a plantation forest tree for the production of wood and fiber and as a cellulose feedstock for biofuel production. In this larger context of poplar, both as a model and applied system, many questions fundamental to tree biology, such as lignin and cellulose formation, perennial growth, dormancy, and resistance against biotic and abiotic stress are being addressed using genomic approaches (Andersson et al. 2004; Andersson-Gunnerås et al. 2006; Brosché et al. 2005; Gu et al. 2004; Hertzberg et al. 2001; Kohler et al. 2003; Major and Constabel 2006; Ralph et al. 2006; Smith et al. 2004).

In their natural environment, poplars are often keystone species in temperate ecosystems, in which they interact with a diverse array of insect pests, pathogens, or symbionts (Arimura et al. 2004; Major and Constabel 2006; Ralph et al. 2006; Smith et al. 2004). With the large-scale deployment of poplar in plantation forestry and with changing environments, pressure from pathogens, herbivores, and abiotic stress will likely increase and could offset many of the possible benefits of poplar as a rapidly renewable resource for biomaterials. The Populus system is therefore an important system for addressing eco- 
logical questions, including the interactions of poplar with other organisms and adaptation to the environment, using genomic approaches.

The interaction of herbaceous plants with pathogens has been extensively investigated using genetic and molecular tools (Nimchuk et al. 2003), but by contrast, our knowledge of defense mechanisms in tree species to pathogens is still largely incomplete. Poplars are hosts for a large variety of pathogens, including fungal biotrophs (Melampsora species), necrotrophs (e.g., Venturia populina), bacteria (e.g., Xanthomonas popular), and viruses (e.g., Poplar mosaic virus) (Callan 1998; Newcombe et al. 1996). One of the most destructive diseases of poplar is Melampsora leaf rust, which causes premature leaf drop, loss of biomass and vigor, and even death in poplar plantations (Newcombe et al. 1994). M. medusae causes the major leaf rust disease of $P$. deltoides found in eastern North America, and the pathogen has now spread to the northwestern U.S., following the introduction of $P$. trichocarpa $\times P$. deltoides (TD) hybrids as plantation crops (Newcombe et al. 1996). In Europe, the leaf rust fungus $M$. larici-populina is a common poplar pathogen (Frey et al. 2005). The alternate hosts for Melampsora poplar rust fungi are usually conifers, and infection of poplar leaves in the spring occurs from conifer-produced aeciospores (Callan 1998). On infected poplar leaves, asexual urediospores can be produced several times within a season, so the spread of rust can proceed rapidly under moist conditions. Although resistance to $M$. medusae in TD hybrids is heritable and major resistance genes have been mapped (Newcombe et al. 1996; Yin et al. 2004), it is not known how this resistance is mediated at the biochemical level.

Plant-pathogen defense is an active process and is associated with altered expression levels of up to $20 \%$ of genes within the host plant genome (Nimchuk et al. 2003). Genes typically activated by pathogens are involved in cell-wall modifications or encode antimicrobial proteins such as plant defensins, thionins, $\beta$-glucanases, and chitinases (Chisholm et al. 2006; Heath 2000; Schenk et al. 2000). Many upregulated genes encode socalled pathogenesis-related (PR) proteins, which are considered hallmarks of an active defense. PR proteins have been described in many species and, in some pathosystems, are directly inhibitory to pathogens (Van Loon et al. 2006). Pathogen challenge also often induces genes encoding enzymes required for biosynthesis of phytoalexins. These antimicrobial secondary metabolites can be derived from phenylpropanoid, terpenoid, or amino acid pathways (Dixon 2001). The plant defense response also includes the activation of a variety of regulatory genes and proteins including transcription factors and protein kinases (Eulgem 2005; Nimchuk et al. 2003; Schenk et al. 2003).

The dramatic pathogen-induced alteration in transcriptional activity has made studying the transcriptome during infection or resistance an effective strategy for probing plant defense. DNA microarray analysis in Arabidopsis has documented the
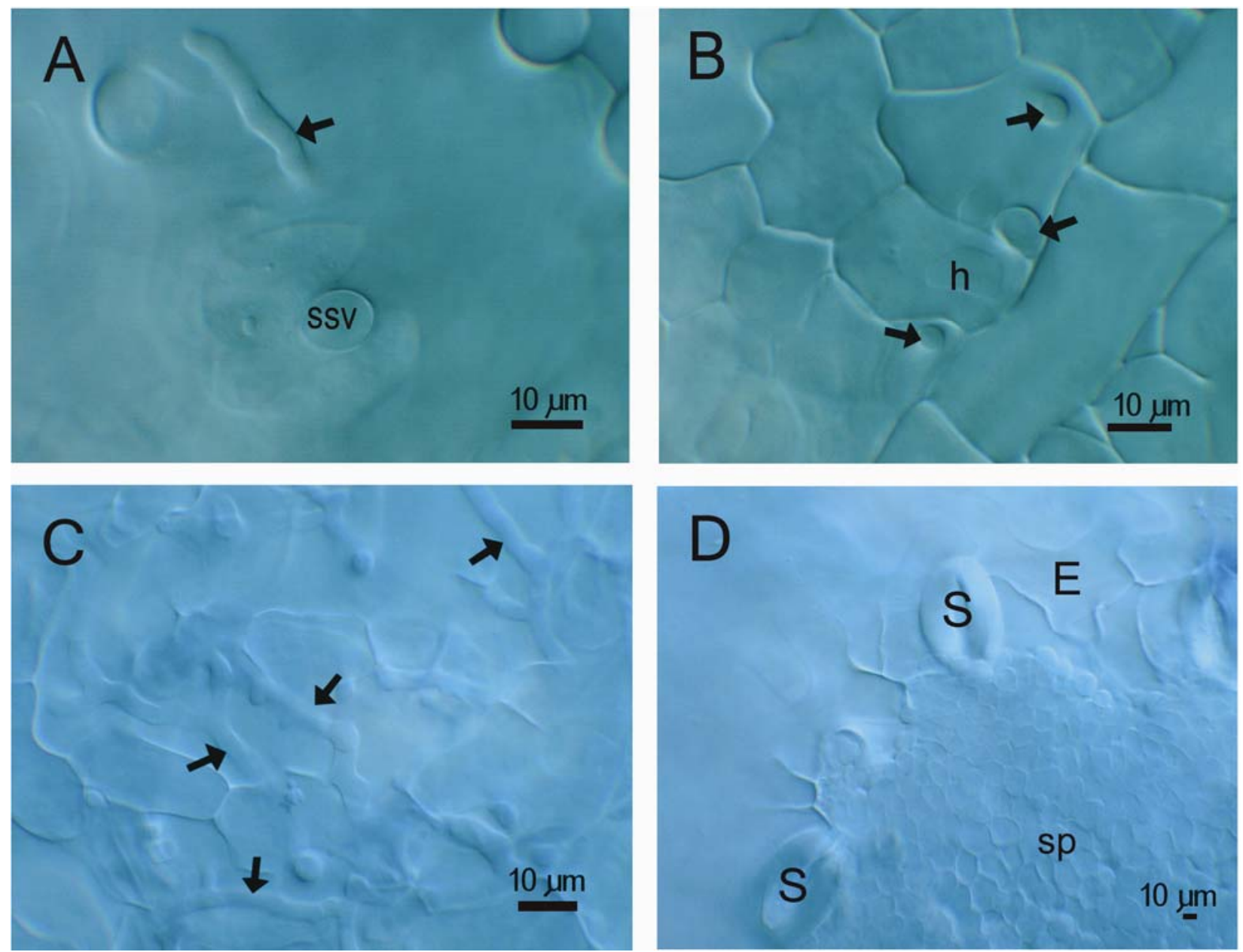

Fig. 1. Light micrographs of infection sites of Melampsora medusae in whole, cleared poplar leaves. A, A secondary "runner" hypha (arrow) just under the epidermis at 1 day postinoculation (dpi). The fungal substomatal vesicle (ssv) is visible between the guard cells of the stoma. B, Optical cross sections of several intercellular hyphae (arrows) and an intracellular haustorium (h) in a 2-day-old fungal colony. C, Extensive intercellular hyphae (arrows) in a 3-dayold colony. D, Hyphal mass that is beginning to form urediospores (sp) just under the epidermis at 6 dpi. The fungal mass raises the epidermis so that stomata (S) and plant epidermal cells (E) can be seen in the same focal plane as the fungus. 
upregulation of hundreds to thousands of genes following pathogen attack (Glazebrook et al. 2003; Maleck et al. 2000; Moy et al. 2004; Schenk et al. 2000, 2003; Tao et al. 2003; Thilmony et al. 2006). Microarrays are thus potent tools for identifying novel defense functions or mechanisms from patterns of gene expression (Restrepo et al. 2005). This general approach of defense gene discovery has been successful in both compatible (susceptible plants) and incompatible (resistant plants) plantpathogen interactions, since many of the same genes are expressed in both situations but differ quantitatively and in timing (Katagiri 2004; Tao et al. 2003; Thilmony et al. 2006). For instance, the successful infection of soybean with Phytophthora sojae leads to the expression of defense genes involved in resistance, including genes encoding PR proteins and phytoalexin enzymes (Moy et al. 2004). Applying gene expression profiling to compatible plant-pathogen interactions may also provide clues as to how biotrophic pathogens escape detection by the host plant or interfere with host defenses and therefore prevent the hypersensitive response and cell death. These processes are poorly understood at present for interactions of plants with biotrophic fungi, in part because no compatible rust fungus is available for Arabidopsis (Mellersh and Heath 2003) but are likely of great importance in the evolution of specialized biotrophic pathogens such as the rust fungi (O'Connell and Panstruga 2006; Voegele and Mendgen 2003).

In this report, we describe experiments that aim to profile the transcriptional response of hybrid poplar leaves in a compatible interaction with the biotrophic rust fungus $M$. medusae, using a poplar cDNA microarray containing 15,496 elements (Ralph et al. 2006). Our results indicate that the poplar leaf transcriptome is dramatically altered by infection, with the majority of changes in transcript abundance occurring late in the infection (6 to 9 dpi). In a comparative analysis with insect-induced defenses in leaves of the same hybrid poplar (Ralph et al. 2006), we found that M. medusae infection downregulates many of the major herbivore-inducible defenses. $M$. medusae rust infection strongly induced genes for enzymes of flavonoid and proanthocyanidin (condensed tannin) biosynthesis late in the infection process, linking this pathway for the first time to the pathogen defense response in poplar. The latter result was confirmed by induced levels of proanthocyanidins in $M$. medusae-infected leaves.

\section{RESULTS}

\section{The $M$. medusae-hybrid poplar interaction}

\section{is highly compatible and involves rapid hyphal growth.}

To investigate defense mechanisms of hybrid poplar to Melampsora leaf rust infection, we inoculated a susceptible $P$. trichocarpa $\times P$. deltoides hybrid (genotype H11-11) with urediospores of $M$. medusae. The leaf-rust pathogen infects and reproduces readily on this poplar hybrid and produces uredinial pustules containing asexual urediospores 8 to 10 days postinoculation (dpi). Preliminary Northern analysis showed that PR-1 and phenylalanine ammonia lyase (PAL) transcripts were upregulated as early as $1 \mathrm{dpi}$, indicating that at least some recognition of the pathogen had occurred and some responses had been triggered (data not shown). However, no macroscopic symptoms were visible on the plants prior to $4 \mathrm{dpi}$.

To determine the extent of pathogen growth prior to development of macroscopic symptoms, we examined inoculated leaves by interference-contrast microscopy. At 1 dpi, we detected infection sites within the leaf (Fig. 1A). These sites varied from having only substomatal vesicles and short intercellular infection hyphae to other sites with long infection hyphae and secondary hyphae totaling over $100 \mu \mathrm{m}$ in length. Some secondary hyphae grew just beneath the epidermis as "runner" hyphae (Fig. 1A) and most infection sites had at least one large haustorium (Fig. 1B). By 2 dpi, at most infection sites, a small fungal colony with about five large haustoria in the central region had developed, with a few smaller haustoria associated with the long runner hyphae below the epidermis (not shown). At 3 dpi, fungal growth was extensive within each colony (Fig. 1C), and at $6 \mathrm{dpi}$, hyphal masses that were beginning to form urediospores could be seen just under the epidermis at each infection site (Fig. 1D). By 9 dpi, mature uredia had formed and begun breaking through the epidermis and releasing urediospores (not shown). The interaction between this M. medusae isolate and the H11-11 hybrid appeared to be highly compatible, since we observed no visible evidence in any cells of defensive callose or phenolic depositions in cell walls or surrounding haustoria.

\section{M. medusae infection of hybrid poplar leaves triggers large changes in gene expression late in the infection.}

Based on these histological observations and the initial Northern analysis, we designed timecourse studies for gene expression profiling using a poplar cDNA microarray platform that contained 15,496 cDNA elements from a variety of EST libraries (Ralph et al. 2006). Infected and control leaves were harvested at 1, 3, 6, and 9 dpi for RNA isolation, cDNA synthesis, and microarray hybridization. For each timepoint, equal amounts of pooled total RNA from five plants (infected or control) was hybridized pairwise in four technical replicates to determine differential gene expression. We had previously used the same poplar microarray platform for an analysis of gene expression of the same clonal poplar hybrid genotype in response to feeding by forest tent caterpillar larvae Malacosoma disstria (Ralph et al. 2006). In that study, using rigorous statistical testing of biological and technical replicates, we demonstrated that almost all observed variation in microarray signals was due to technical rather than biological variation when clonal trees were used under greenhouse conditions (Ralph et al. 2006). We therefore applied the same protocol of array analysis for the present study followed by targeted validation of transcript abundance (discussed below). Array elements displaying a $1.5 \times$ fold change between infected and control leaves with $P<0.05$ and $Q=<0.10$ were considered to be differentially expressed (DE). At a threshold of $P=0.05$, false discovery rates were estimated to be $9.1,8.6,0.6$, and $0.9 \%$ for the 1 , 3, 6, and 9 dpi timepoints, respectively, based on $Q$ values (Storey and Tibshirani 2003). Using these statistical criteria, nearly $20 \%$ of elements on the array were DE at one or more timepoints in infected leaves. A total of 1,388 elements $(9.0 \%)$ were upregulated (induced), while 1,404 elements $(9.1 \%)$ were downregulated (repressed) for at least one timepoint (Fig. 2). Supplemental Table 1 provides a complete list of expression data for all genes represented on the microarray.

To gain further insight into the infection process, we analyzed the temporal patterns of up- and downregulated transcript abundance (Fig. 2A). At 1 and 3 dpi, only 72 and 63 elements were induced, respectively, while at the 6 and 9 dpi timepoints, 1,023 and 613 were upregulated, respectively. The number of correspondingly downregulated elements at the 1,3 , and 6 dpi timepoints was slightly lower (22, 48, and 921 elements, respectively), whereas at 9 dpi the number of repressed genes was greater than the number induced (779 elements; Fig. 2A). Thus, there was a dramatic increase in the number of DE genes between 3 and 6 dpi, suggesting a substantial change in the infection process and host response. We visualized the overlap in induced gene sets for the different timepoints using a Venn diagram (Fig. 2B), which confirmed the prominent shift in gene expression between 3 and 6 dpi. While $64 \%$ (46 of 72) and 70\% (718 of 1,023) of the elements induced at 1 and 6 dpi, respec- 
tively, were exclusively upregulated at these timepoints, only $17 \%$ (11 of 63 ) of the elements induced at 3 dpi were uniquely expressed (Fig. 2B). The 3 dpi timepoint may, therefore, be in the transition phase between early and late responses. Interest- ingly, almost one-half of the genes significantly repressed at 3 dpi were also induced at 6 dpi (Supplemental Table 2), emphasizing this transition and perhaps indicating a release of general repression. The overlap in induced genes at the 6 and 9 dpi time-
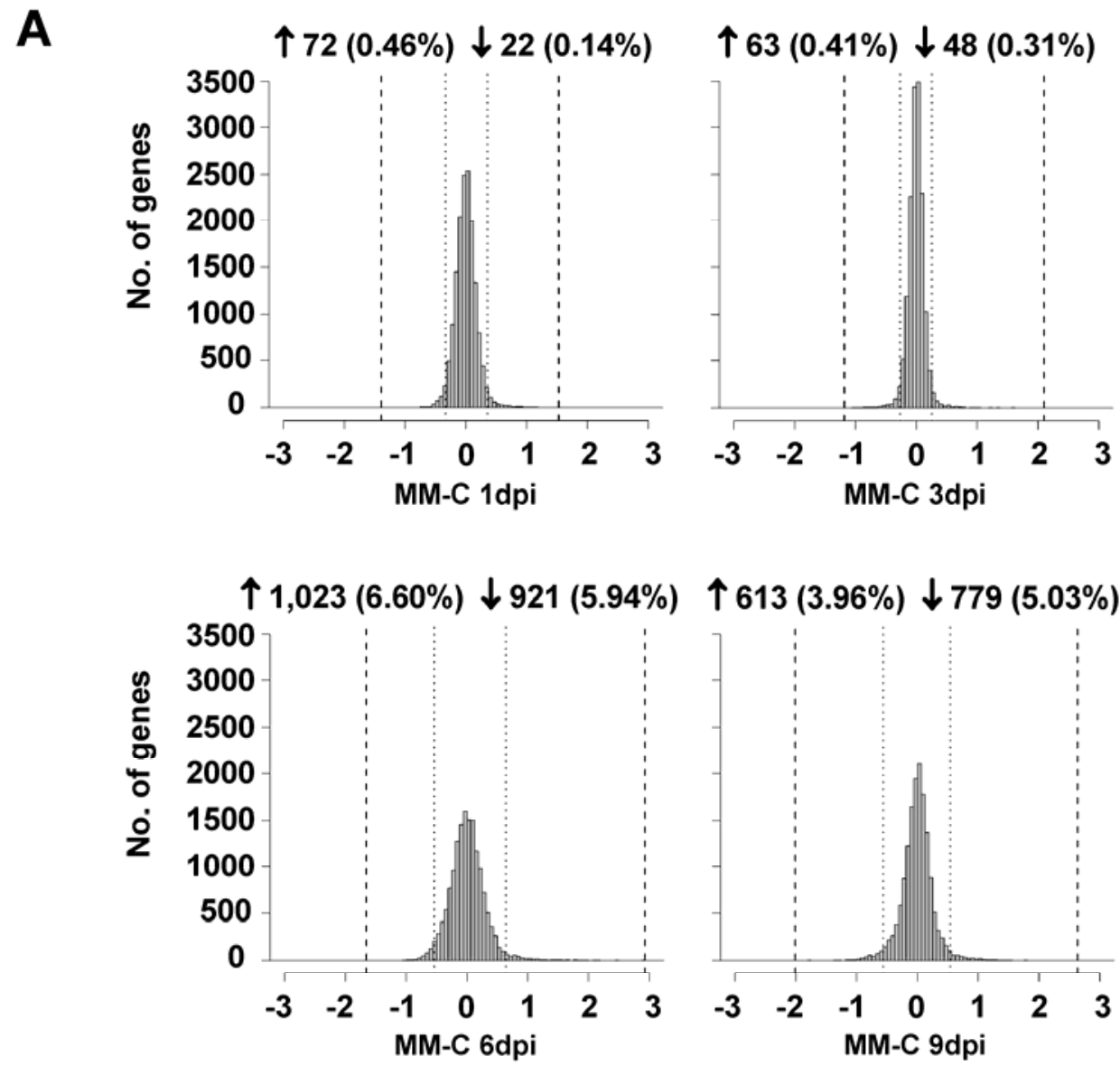

Fold change (natural log)

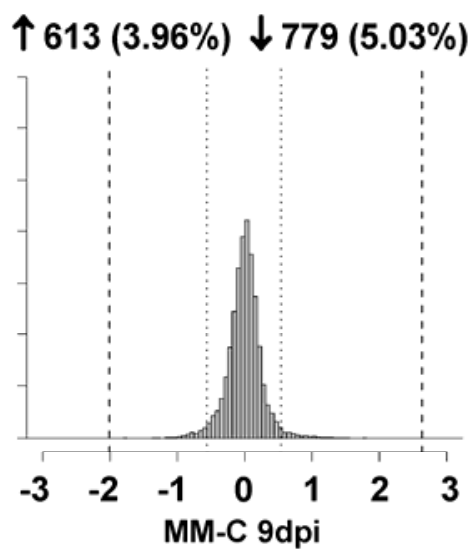

Fold change (natural log)

B

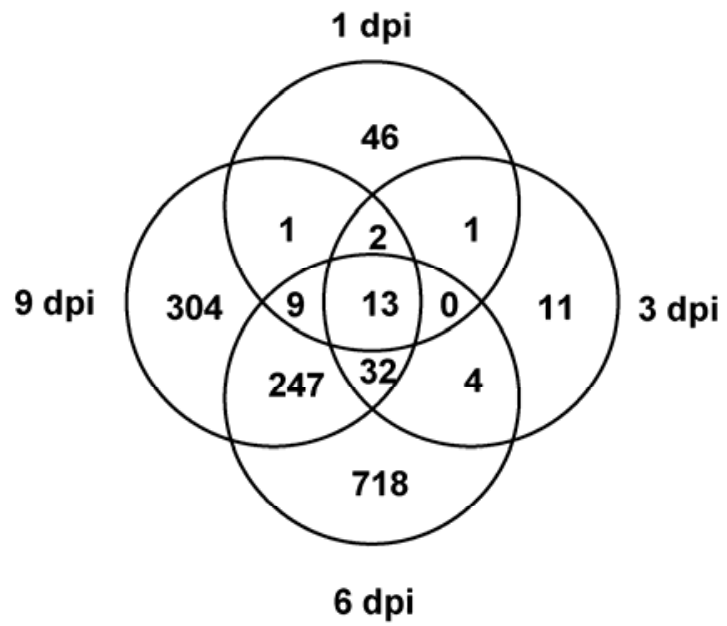

Downregulation

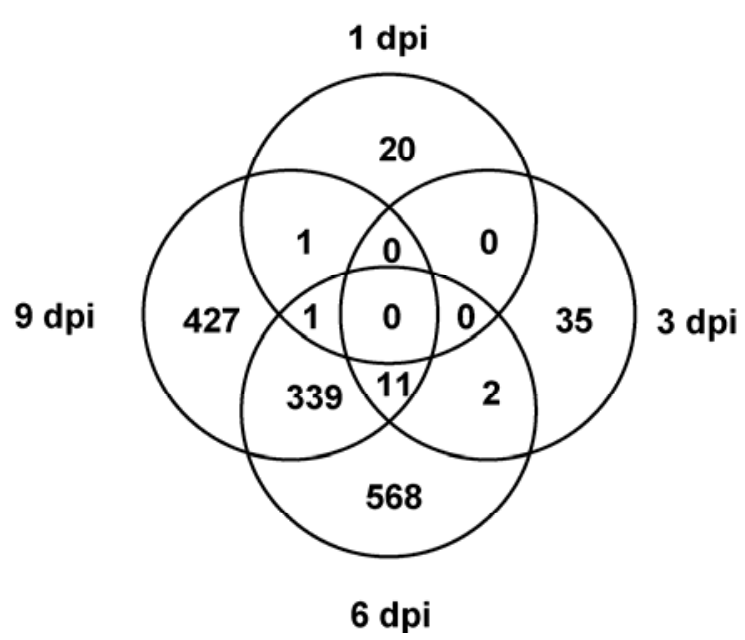

Fig. 2. Summary of differentially expressed (DE) genes from microarray analysis of 15,496 array elements during infection of poplar leaves with Melampsora medusae (MM). Genes were classified as DE between infected and control (C) leaves using a threshold of fold change $>1.5, P<0.05$ and $Q<0.10$. A, Histograms of the fold change (MM-C) distribution for all genes at 1,3,6, and 9 days postinoculation (dpi). Short-dash vertical bars indicate the upper and lower $95 \%$ confidence intervals and long-dash bars indicate maximum up- or downregulation. The number of genes up- or downregulated are indicated for each timepoint, with numbers in parenthesis representing DE genes as a percentage. B, Venn diagram showing the overlap of up- or downregulated elements between the various timepoints. 
points was substantial, indicating an activated defense response at these late timepoints. Similar patterns of overlap were observed for the repressed gene sets.

Despite the substantial number of induced genes, few (13 of $1,388)$ were upregulated significantly and none were downregulated at all timepoints (Fig. 2B). Thus, distinct sets of genes are affected at different stages of the infection. Of the genes in the common induced set, a large proportion are annotated as encoding PR proteins such as PR-1, endochitinases, glucanases, and dirigent proteins (Table 1). PR-1 and other PR proteins are found in many if not all plants, and PR-1 is considered to be a robust marker for the induced defense response (Van Loon et al. 2006). In addition to these known defense genes, genes encoding ADP-ribosylation factor ARF-1, several Myb family transcription factors, a senescence-related protein, and a number of unknown proteins are also upregulated throughout the entire timecourse.

\section{Many Melampsora-repressed genes encode photosynthesis-related and herbivore-inducible defense proteins.}

To further discern global trends and identify patterns of coexpressed genes in the infection process, we used the divisive DIANA algorithm (Bryan 2004) for cluster analysis (Fig. 3). Eight unique clusters showing distinct profiles were generated, collectively representing gene sets exclusively with up- or downregulation and gene sets with mixed response patterns (Fig. 3). Clusters 1 to 5 contained the majority of DE genes on the array and generally demonstrated profiles of consistent upor downregulation. Clusters 6 to 8 contained fewer genes, but they showed stronger changes in expression. We also exam- ined clusters for functional annotation patterns and found that most clusters typically contained genes belonging to diverse functional categories.

We first focused on clusters that grouped genes with decreasing expression, as the biotrophic nature of $M$. medusae and a lack of macroscopic symptoms and major induction of gene expression until at least 3 dpi (Fig. 2) suggests that early suppression of defense could be important for successful colonization. Despite extensive screening for genes with a pattern of very early repression, such genes were not exclusively represented as a unique cluster. While genes in cluster 6 were among the most strongly downregulated at $1 \mathrm{dpi}$, expression ratios were still quite variable at $1 \mathrm{dpi}$ and not statistically significant. In contrast to cluster 6 , cluster 3 grouped genes that are first upregulated and then progressively repressed during the timecourse after 3 dpi (Fig. 3). Among the most strongly repressed genes identified at 9 dpi in cluster 3 were many photosynthesis-related genes, including chlorophyll $\mathrm{a} / \mathrm{b}$ binding proteins and photosynthetic reaction center proteins. Other elements repressed at 6 and 9 dpi in cluster 3 included many genes involved in carbon metabolism (such as fructose-bisphosphate aldolase, sugar isomerase, sucrose synthase), suggesting an overall downregulation of primary processes as a defense response is activated at the later time of successful infection. No PR or other known defense-related genes were found in this set.

Similar to those in cluster 3 , genes from cluster 7 also showed induction early in the infection process; however, in contrast, these genes then followed a much stronger general decline in transcript abundance, with lowest levels observed at 9 dpi (Fig. 3). Surprisingly, this cluster contained a substantial number of

Table 1. Microarray elements upregulated at all timepoints following infection with Melampsora medusae ${ }^{\mathrm{a}}$

\begin{tabular}{|c|c|c|c|c|c|c|c|c|c|c|c|}
\hline \multirow[b]{2}{*}{ Clone ID } & \multirow[b]{2}{*}{ AGI number } & \multirow[b]{2}{*}{ E-value } & \multirow[b]{2}{*}{ Annotation $^{b}$} & \multicolumn{2}{|c|}{1 dpi } & \multicolumn{2}{|c|}{3 dpi } & \multicolumn{2}{|c|}{6 dpi } & \multicolumn{2}{|c|}{9 dpi } \\
\hline & & & & FC & $\boldsymbol{P}$ & FC & $\boldsymbol{P}$ & FC & $\boldsymbol{P}$ & FC & $\boldsymbol{P}$ \\
\hline WS0173_N04 & At1g64160 & $1 e-30$ & $\begin{array}{l}\text { Disease resistance-responsive/dirigent } \\
\text { protein }\end{array}$ & 1.87 & 0.002 & 2.70 & $<0.001$ & 6.13 & $<0.001$ & 3.17 & $<0.001$ \\
\hline WS0147_I21 & At2g14580 & $3 e-53$ & Pathogenesis-related protein 1 (PR-1) & 2.00 & $<0.001$ & 3.53 & $<0.001$ & 2.45 & $<0.001$ & 13.92 & $<0.001$ \\
\hline WS0224_B11 & At2g14610 & $3 e-57$ & Pathogenesis-related protein 1 (PR-1) & 1.67 & 0.019 & 2.48 & $<0.001$ & 2.15 & 0.002 & 3.61 & $<0.001$ \\
\hline WS0175_A23 & At2g47170 & $1 e-97$ & ADP-ribosylation factor 1 (ARF1) & 3.19 & $<0.001$ & 8.17 & $<0.001$ & 5.23 & $<0.001$ & 7.48 & $<0.001$ \\
\hline WS0145_K24 & At3g03080 & $4 e-21$ & NADP-dependent oxidoreductase & 1.86 & 0.034 & 3.35 & 0.001 & 7.02 & $<0.001$ & 9.11 & $<0.001$ \\
\hline WS01211_N10 & At3g07130 & $2 \mathrm{e}-73$ & Serine/threonine protein phosphatase & 1.64 & $<0.001$ & 2.29 & $<0.001$ & 2.40 & $<0.001$ & 1.86 & $<0.001$ \\
\hline WS0161_Ē14 & At3g10740 & $3 e-19$ & Glycosyl hydrolase & 3.04 & $<0.001$ & 1.71 & 0.005 & 3.07 & $<0.001$ & 3.94 & $<0.001$ \\
\hline WS0209_J01 & At3g12500 & $3 e-34$ & Basic endochitinase & 1.77 & 0.004 & 2.49 & $<0.001$ & 2.36 & $<0.001$ & 3.52 & $<0.001$ \\
\hline WS0162_K02 & At3g45600 & $6 e-45$ & Senescence-associated protein & 2.04 & $<0.001$ & 2.81 & $<0.001$ & 5.14 & $<0.001$ & 6.04 & $<0.001$ \\
\hline WS0124_E20 & At3g47540 & $8 \mathrm{e}-11$ & Basic endochitinase & 1.50 & 0.020 & 2.03 & 0.001 & 1.84 & 0.002 & 2.82 & $<0.001$ \\
\hline WS01218_H13 & At3g55990 & $7 e-09$ & Expressed protein & 2.53 & $<0.001$ & 1.92 & 0.001 & 2.20 & $<0.001$ & 2.67 & $<0.001$ \\
\hline WS0122_H18 & At3g57260 & $2 \mathrm{e}-34$ & $\begin{array}{l}\text { Glucan endo-1,3-beta-glucosidase, acidic } \\
\text { isoform }\end{array}$ & 1.95 & 0.001 & 3.88 & $<0.001$ & 4.46 & $<0.001$ & 6.63 & $<0.001$ \\
\hline WS0132_L23 & At3g57270 & $7 e-49$ & Beta-1,3-glucanase & 1.69 & $<0.001$ & 3.34 & $<0.001$ & 5.77 & $<0.001$ & 5.57 & $<0.001$ \\
\hline WS0181_F07 & At4g37260 & $2 \mathrm{e}-40$ & Myb family transcription factor (MYB73) & 1.97 & $<0.001$ & 3.29 & $<0.001$ & 2.74 & $<0.001$ & 8.04 & $<0.001$ \\
\hline WS0175_O01 & At4g38620 & $2 \mathrm{e}-12$ & Myb family transcription factor (MYB4) & 2.05 & 0.003 & 4.98 & $<0.001$ & 2.61 & $<0.001$ & 11.35 & $<0.001$ \\
\hline WS01211_NN17 & At5g09600 & $1 \mathrm{e}-15$ & Succinate dehydrogenase subunit 3 & 1.77 & $<0.001$ & 2.78 & $<0.001$ & 1.88 & $<0.001$ & 1.88 & $<0.001$ \\
\hline WS01119_K08 & At5g10360 & $3 e-76$ & $40 \mathrm{~S}$ ribosomal protein & 3.30 & $<0.001$ & 1.68 & $<0.001$ & 4.54 & $<0.001$ & 2.87 & $<0.001$ \\
\hline WS0204_B10 & At5g13220 & $2 \mathrm{e}-18$ & Expressed protein & 1.52 & 0.005 & 2.87 & $<0.001$ & 2.29 & $<0.001$ & 2.45 & $<0.001$ \\
\hline WS0162_M02 & At5g66930 & $5 e-73$ & Expressed protein & 1.80 & $<0.001$ & 4.33 & $<0.001$ & 2.97 & $<0.001$ & 6.01 & $<0.001$ \\
\hline WS0213_B15 & n.a. & n.a. & No significant hit & 2.63 & $<0.001$ & 4.84 & $<0.001$ & 8.10 & $<0.001$ & 8.58 & $<0.001$ \\
\hline WS0207_E14 & n.a. & n.a. & No significant hit & 1.53 & 0.007 & 4.08 & $<0.001$ & 3.54 & $<0.001$ & 2.46 & $<0.001$ \\
\hline WS0162_J02 & n.a. & n.a. & No significant hit & 2.07 & 0.024 & 3.80 & 0.001 & 8.49 & $<0.001$ & 6.22 & $<0.001$ \\
\hline WS0127_F14 & n.a. & n.a. & No significant hit & 1.88 & 0.001 & 2.40 & $<0.001$ & 1.95 & $<0.001$ & 3.00 & 0.001 \\
\hline WS0147_H21 & n.a. & n.a. & No significant hit & 1.78 & 0.001 & 2.17 & $<0.001$ & 2.14 & 0.001 & 1.82 & $<0.001$ \\
\hline WS0211_H11 & n.a. & n.a. & No significant hit & 3.61 & $<0.001$ & 2.15 & $<0.001$ & 3.23 & $<0.001$ & 4.54 & $<0.001$ \\
\hline WS0122_O18 & n.a. & n.a. & No significant hit & 1.56 & 0.013 & 2.14 & $<0.001$ & 2.19 & $<0.001$ & 1.83 & 0.002 \\
\hline WS0153_D07 & n.a. & n.a. & No significant hit & 2.90 & $<0.001$ & 1.99 & $<0.001$ & 3.19 & $<0.001$ & 2.27 & $<0.001$ \\
\hline WS0145_C05 & n.a. & n.a. & No significant hit & 1.59 & 0.003 & 1.92 & $<0.001$ & 1.81 & 0.001 & 1.68 & 0.002 \\
\hline
\end{tabular}

${ }^{a}$ Elements with a fold-change (FC) $>1.5 \times$ (infected/control leaves) and $P<0.05$ at all time points, with $Q<0.10$ for at least three time points. Genes in bold indicate known pathogen defense-related genes. dpi = days postinoculation; AGI = Arabidopsis Gene Index.

${ }^{\mathrm{b}}$ BLASTX vs. Arabidopsis putative annotation of array elements assigned according to The Arabidopsis Information Resource protein set using BLASTX with a threshold of expect $(\mathrm{E})$ value $<1 \mathrm{e}-05$. 
genes that previous work had identified as strongly wound- and forest tent caterpillar-inducible defense genes (Major and Constabel 2006; Ralph et al. 2006). Therefore, we further compared expression data for $M$. medusae infection with data obtained from another experiment in which the same array platform and same poplar genotype were used to probe herbivoreinduced gene expression (S. Ralph and J. Bohlmann, unpublished). Table 2 lists expression ratios for genes highly repressed by $M$. medusae infection at $9 \mathrm{dpi}$, together with corresponding gene expression in leaves following forest tent caterpillar herbivory. Surprisingly, many genes repressed by Melampsora infection at 3,6, and 9 dpi were conversely induced $24 \mathrm{~h}$ after continuous herbivory, though often weakly downregulated at $2 \mathrm{~h}$. The genes on this list encode proteins that are well characterized in poplar herbivore interactions such as Kunitz trypsin inhibitors (TI), chitinases, polyphenol oxidase (PPO), a terpene synthase, and other herbivore defense genes (Christopher et al. 2004; Major and Constabel 2006; Ralph et al. 2006). In general, genes with the lowest expression ratios at $9 \mathrm{dpi}$ in the interaction with $M$. medusae were among the most strongly caterpillar-induced genes (Table 2), indicative of a largely divergent and possibly antagonistic response of poplar to a biotrophic fungal pathogen and insect feeding.

\section{Strongly Melampsora-induced genes encode PR proteins and enzymes required for flavonoid biosynthesis.}

Clusters 1 and 4 contain genes with broad upward trends in transcript abundance following infection (Fig. 3). Interestingly, among the 50 most strongly induced genes in cluster 4 at 9 dpi, $30 \%$ are annotated for PR or cell wall-modifying functions. Across all clusters, these categories comprise approximately $20 \%$ of the 100 most strongly induced genes at 9 dpi. Cluster 1 comprises many genes with peak induction at $6 \mathrm{dpi}$ (Fig. 3) and includes several flavonoid biosynthetic as well as PR genes. Cluster 6 also contains genes that are upregulated strongly at 6 dpi following downregulation early in the infection, with a large proportion $(22 \%)$ of genes annotated for flavonoid and phenylpropanoid metabolism.

To highlight individual genes induced over the course of infection, Table 3 presents the 15 most highly induced array elements for each timepoint. At $1 \mathrm{dpi}$, at least one known herbivore-responsive gene (a Kunitz TI) shows strong induction, which suggests that the pathogen is triggering at least a partial wound response despite the lack of physical indications of an attempted defense or containment of the fungus. Although many herbivory defense genes are ultimately repressed at later timepoints, during the early response some herbivore and pathogen response genes are upregulated in parallel. At $3 \mathrm{dpi}$, genes potentially involved in pathogen responses (i.e., PR-1, chitinases, $\beta$-1,3-glucanases, WRKY transcription factors) are well represented, accounting for 13 of 54 elements with twofold or greater induction (Table 3). As early as $3 \mathrm{dpi}$, the induction of flavonoid biosynthetic genes is evident (e.g., flavanone 3-hydroxylase $[\mathrm{F} 3 \mathrm{H}]$ ), and this is even more pronounced at $6 \mathrm{dpi}$ (discussed below). At $9 \mathrm{dpi}, 11$ of the top 50 induced genes are annotated for pathogen defense, together with genes associated with a broad range of other biological functions (Table 3).

The most pronounced response to $M$. medusae infection of poplar leaves, both in terms of the number of genes and magnitude of induction, occurred at $6 \mathrm{dpi}$. Among array elements significantly upregulated fivefold or more, almost one-third are annotated as flavonoid-related genes, and six of these rank in the top 15 (Table 3). The dramatic rise in DE genes between 3 and 6 dpi (Fig. 2) appears to involve genes for general phenolic secondary metabolism, several of which were initially downregulated at 1 and 3 dpi. Most flavonoid biosynthesis genes, however, showed unaltered gene expression at the earlier timepoints, emphasizing the dramatic switch in metabolism at $6 \mathrm{dpi}$. The list of strongly induced genes at 6 dpi included chalcone synthase, $\mathrm{F} 3 \mathrm{H}$, and leuco-
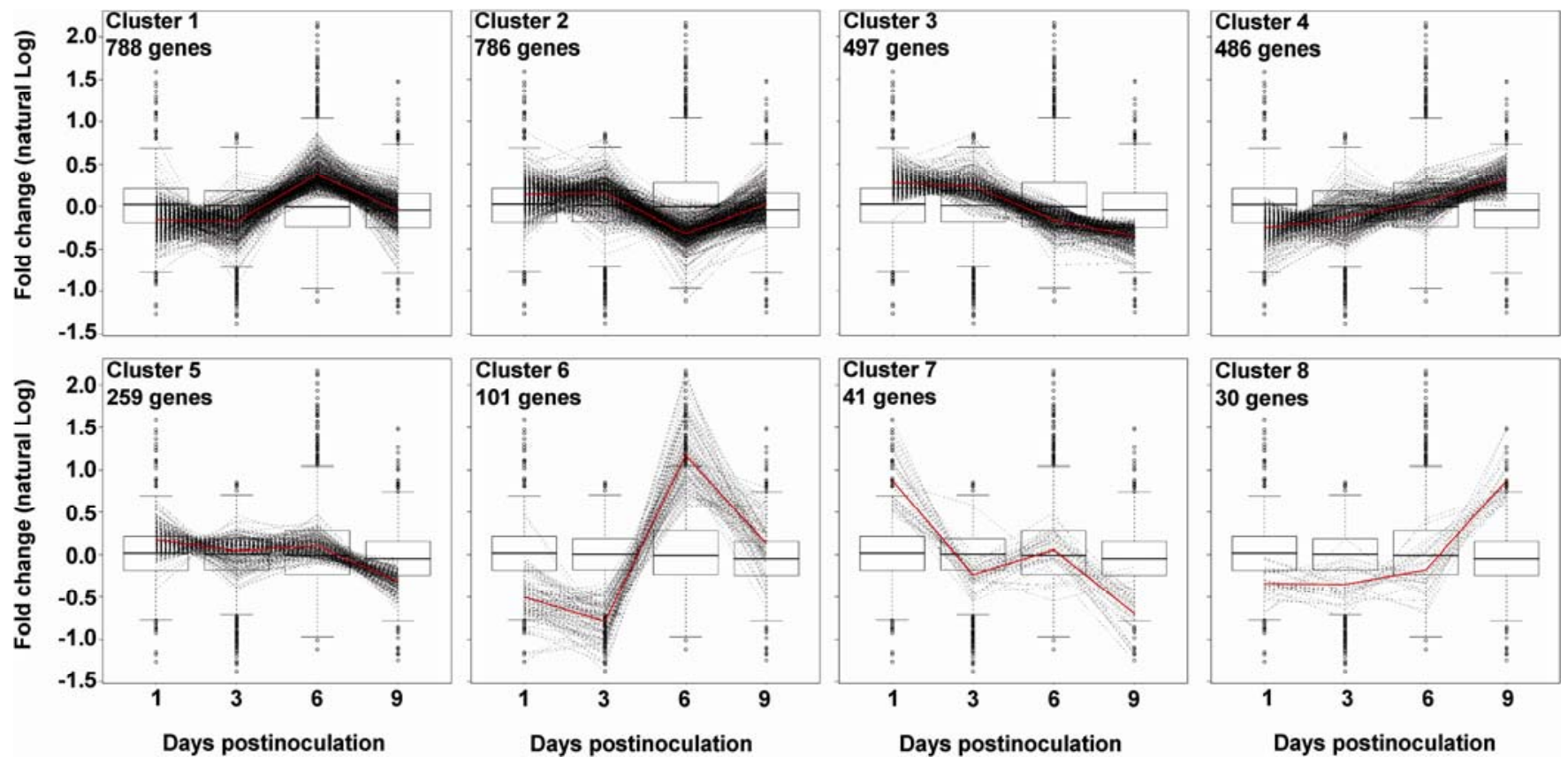

Fig. 3. Cluster analysis of expression profiles of genes differentially expressed (DE) following Melampsora medusae infection of poplar leaves. A set of 2,988 genes were identified as DE (fold change $>1.5$ for infected vs. control leaves; $P<0.05$ ) for at least one timepoint and then were clustered using the divisive DIANA algorithm (Bryan 2004). The first eight clusters formed were examined. For each panel, fold change expression ratios are plotted for 1, 3,6 , and 9 days postinoculation. Black dotted lines represent the expression profiles of individual array elements, and red lines represent the median expression ratio for an entire cluster. For each timepoint, boxplot representations of the expression profile for all 2,988 array elements are provided for reference. Each boxplot shows the median value as a line bisecting the box, upper (75\%) and lower (25\%) quartiles at the top and bottom box edges, the nonoutlier minimum and maximum values as whiskers outside the box ( $1.5 \times$ the interquartile range), and outlier values (beyond the whiskers) as open circles. 
anthocyanidin dioxygenase (also referred to as anthocyanidin synthase) (Table 3). Genes from the early steps of phenylpropanoid metabolism, such as PAL and 4-coumarate-CoA ligase (4CL) were also induced at 6 dpi (5- and 3.5-fold induction, respectively). Likewise, genes encoding shikimate pathway enzymes for the biosynthesis of aromatic amino acids were induced severalfold, for example, 3-deoxy D-arabinoheptulosonate 7-phosphate (DAHP) synthase. These results suggest $M$. medusae infection induces both the shikimate and general phenylpropanoid pathways as well as flavonoid biosynthesis, which is downstream of these early steps in phenylpropanoid biosynthesis.

\section{M. medusae infection induces \\ proanthocyanidin biosynthesis in poplar leaves.}

The flavonoid pathway comprises several branches, which together lead to a large diversity of secondary metabolites including anthocyanins, flavonols, flavones, isoflavonoids, and

Table 2. Comparison of changes in gene expression following Melampsora medusae infection and forest tent caterpillar (FTC, Malacosoma disstria) feeding ${ }^{\mathrm{a}}$

\begin{tabular}{|c|c|c|c|c|c|c|c|c|c|c|c|c|c|c|c|}
\hline \multirow[b]{3}{*}{ Clone ID } & \multirow[b]{3}{*}{ AGI number } & \multirow[b]{3}{*}{ E-value } & \multirow[b]{3}{*}{ BLASTX vs. Arabidopsis ${ }^{\mathrm{b}}$} & \multicolumn{8}{|c|}{ M. medusae infection } & \multicolumn{4}{|c|}{ FTC herbivory } \\
\hline & & & & \multicolumn{2}{|c|}{$1 \mathrm{dpi}$} & \multicolumn{2}{|c|}{3 dpi } & \multicolumn{2}{|c|}{6 dpi } & \multicolumn{2}{|c|}{9 dpi } & \multicolumn{2}{|c|}{$2 \mathrm{~h}$} & \multicolumn{2}{|c|}{$24 \mathrm{~h}$} \\
\hline & & & & FC & $P$ & $\mathrm{FC}$ & $P$ & FC & $P$ & FC & $P$ & $\mathrm{FC}$ & $P$ & FC & $P$ \\
\hline WS0212_I21 & At4g33090 & $3 e-66$ & Aminopeptidase & 1.34 & 0.083 & 0.46 & $<0.001$ & 0.55 & 0.003 & 0.16 & $<0.001$ & 0.47 & $<0.001$ & 15.72 & $<0.001$ \\
\hline WS0146_L23 & & $3 e-29$ & Phosphorylase & 2.24 & $<0.001$ & 0.40 & $<0.001$ & 0.56 & $<0.001$ & 0.17 & $<0.001$ & 0.37 & $<0.001$ & 14.55 & $<0.001$ \\
\hline WS0133_I11 & & $3 e-06$ & Kunitz trypsin i & 2.86 & $<0.001$ & 0.36 & $<0.001$ & 0.61 & 0.001 & 0.18 & $<0.001$ & 0.48 & $<0.001$ & 17.47 & $<0.001$ \\
\hline WS01223_D01 & Atlg74320 & $2 \mathrm{e}-80$ & Choline kinase & 2.63 & $<0.001$ & 0.44 & $<0.001$ & 0.58 & $<0.001$ & 0.20 & $<0.001$ & 0.33 & $<0.001$ & 20.32 & $<0.001$ \\
\hline WS0212_O05 & At $2 \mathrm{~g} 24520$ & $3 e-97$ & Plasma membrane ATPase & 1.29 & 0.210 & 0.43 & 0.001 & 0.71 & 0.098 & 0.22 & $<0.001$ & 0.69 & 0.087 & 11.44 & $<0.001$ \\
\hline WS013 & $4 \operatorname{tg} 24340$ & $6 \mathrm{e}-13$ & Phosphorylase & 1.80 & 0.016 & 0.51 & 0.008 & 0.64 & 0.053 & 0.23 & $<0.001$ & 0.37 & $<0.001$ & 14.41 & $<0.001$ \\
\hline $\mathrm{PPO}$ & & & & 1.99 & 0.002 & 0.70 & 0.063 & 0.56 & 0.007 & 0.29 & $<0.001$ & 0.66 & 0.105 & 16.08 & $<0.001$ \\
\hline WS0146_J02 & At3g 12500 & $6 e-25$ & & 1.20 & 0.167 & 0.56 & 0.001 & 0.64 & 0.004 & 0.32 & $<0.001$ & 0.33 & $<0.001$ & 13.05 & $<0.001$ \\
\hline 3_A18 & & & Phos & 1.70 & 0.018 & 0.64 & 0.038 & 0.66 & 0.053 & 0.32 & $<0.001$ & 0.33 & $<0.001$ & 17.40 & $<0.001$ \\
\hline WS02 & At3g12500 & $5 e-10$ & End & 0.90 & 0.624 & 0.55 & 0.013 & 0.67 & 0.075 & 0.34 & $<0.001$ & 0.33 & $<0.001$ & 16.39 & $<0.001$ \\
\hline WS01118_N14 & At5g53300 & $1 e-21$ & Ubiq & 1.39 & 0.046 & 0.59 & 0.004 & 0.85 & 0.292 & 0.34 & $<0.001$ & 0.44 & $<0.001$ & 12.51 & $<0.001$ \\
\hline WS0133_N23 & Atlg & & Kunit & 2.27 & 0.002 & 0.46 & 0.003 & 0.86 & 0.477 & 0.34 & $<0.001$ & 0.47 & $<0.001$ & 11.65 & \\
\hline WS01118_E01 & Atlg04240 & $3 e-35$ & duced protein 3 & 1.88 & 0.001 & 0.39 & $<0.001$ & 0.70 & 0.023 & 0.35 & $<0.001$ & 0.66 & 0.211 & 5.03 & $<0.001$ \\
\hline WS0178_L22 & At5g16800 & & & 1.13 & 0.223 & 0.31 & $<0.001$ & 1.90 & $<0.001$ & 0.37 & $<0.001$ & 0.62 & 0.148 & 3.28 & 0.001 \\
\hline & & & & 1.31 & 0.237 & 0.57 & 0.024 & 0.80 & 0.319 & 0.39 & 0.001 & 0.47 & $<0.001$ & 16.14 & $<0.001$ \\
\hline 18 & 860 & $2 \mathrm{e}-07$ & & 1.68 & 0.056 & 0.55 & 0.032 & 0.69 & 0.150 & 0.39 & 0.003 & 0.62 & 0.006 & 11.61 & $<0.001$ \\
\hline 2_C15 & & $2 e-41$ & & 1.2 & 0.242 & 0.87 & 0.440 & 0.57 & 0.010 & 0.42 & 0.001 & 0.50 & $<0.001$ & 14.02 & $<0.001$ \\
\hline 4_D07 & & & & 0. & 0.895 & 0.84 & 0.110 & 1.86 & $<0.001$ & 0.43 & $<0.001$ & 1.44 & $<0.001$ & 1.66 & \\
\hline WS0124_G12 & At 4 & $3 e-53$ & & 0.98 & 0.758 & 0.58 & $<0.001$ & 1.27 & 0.015 & 0.44 & $<0.001$ & 0.97 & 0.833 & 13.06 & $<0.001$ \\
\hline & & & & & 0.949 & 1.08 & 0.476 & 0.81 & 0.064 & 0.45 & $<0.0$ & 0.97 & 0.930 & 3.91 & \\
\hline & & & & 50 & 0.001 & 0.56 & $<0.001$ & 1.04 & 0.686 & 0.46 & $<0.0$ & 0.50 & $<0.001$ & 28.31 & \\
\hline 5_D20 & 670 & $1 e-47$ & & 0.97 & 0.780 & 0.85 & 0.148 & 0.92 & 0.472 & 0.47 & $<0.0$ & 1.06 & 0.628 & 2.43 & $<0$ \\
\hline WS0151_J12 & & $2 e-28$ & & & 0.140 & 0.44 & 0.002 & 1.15 & 0.509 & 0.47 & 0.003 & 0.36 & $<0.001$ & 13.45 & $<0.0$ \\
\hline WS01120_O24 & At4g07960 & $4 e-13$ & & 1.12 & 0.376 & 0.61 & 0.002 & 0.99 & 0.924 & 0.48 & $<0.001$ & 0.53 & $<0.001$ & 10.10 & $<0.0$ \\
\hline WS0151_F17 & At3g04080 & $4 \mathrm{e}-28$ & & 1. & 0.042 & 0.39 & $<0.001$ & 1.56 & 0.026 & 0.52 & 0.003 & 0.45 & $<0.001$ & 7.44 & $<0.001$ \\
\hline & & & & 1.08 & 0.659 & 0.62 & 0.015 & 0.64 & 0.019 & 0.52 & 0.002 & 0.26 & $<0.001$ & 10.81 & $<0.001$ \\
\hline & & & & 2.03 & $<0.001$ & 0.35 & $<0.001$ & 1.76 & $<0.001$ & 0.52 & $<0.0$ & 0.40 & $<0.001$ & 11.90 & $<0.0$ \\
\hline WS0142_K21 & 2500 & $1 e-55$ & End & 1.28 & 0.027 & 0.85 & 0.126 & 0.91 & 0.344 & 0.54 & $<0.0$ & 0.79 & 0.025 & 7.85 & $<0.001$ \\
\hline WS0133_B09 & At4g15210 & & & & 0.367 & 0.79 & 0.044 & 0.88 & 0.233 & 0.54 & $<0.001$ & 0.68 & $<0.001$ & 3.43 & $<0.001$ \\
\hline WS0174_K18 & At4g33300 & & & 1.07 & 0.342 & 1.02 & 0.835 & 1.29 & 0.004 & 0.54 & $<0.001$ & 0.65 & $<0.001$ & 1.71 & $<0.0$ \\
\hline WSo & & & & & 0.717 & 0.80 & 0.266 & 1.14 & 0.495 & 0.58 & 0.015 & 0.79 & $<0.030$ & 7.30 & $<0$ \\
\hline & & & & 1.02 & 0.874 & 0.90 & 0.362 & 0.57 & $<0.001$ & 0.59 & $<0.001$ & 1.12 & 0.115 & 2.72 & $<0.001$ \\
\hline & At $2 \mathrm{~g} 30080$ & $9 e-51$ & & 1.69 & 0.009 & 0.96 & 0.814 & 0.68 & 0.041 & 0.59 & 0.009 & 0.65 & $<0.001$ & 11.03 & $<0.001$ \\
\hline WS0162_P13 & Atlg77940 & $6 e-18$ & $60 \mathrm{~S} \mathrm{ri}$ & 0.82 & 0.053 & 0.76 & 0.010 & 0.62 & $<0.001$ & 0.60 & $<0.001$ & 1.07 & 0.351 & 1.71 & $<0.001$ \\
\hline WS0132_E12 & At3g12580 & $3 e-78$ & Heat $s$ & 1.23 & 0.158 & 0.89 & 0.399 & 1.76 & 0.002 & 0.60 & 0.003 & 1.41 & $<0.001$ & 1.57 & $<0.001$ \\
\hline WS0156_L05 & At5g59440 & $1 e-58$ & Th & 1.80 & $<0.001$ & 0.76 & 0.042 & 1.27 & 0.073 & 0.60 & 0.001 & 0.82 & 0.232 & 16.56 & $<0.001$ \\
\hline WS & & & & & & 0.49 & & 1.46 & 0.001 & 0.61 & & 0.81 & 0.051 & 3.10 & \\
\hline N 24 & 29420 & $4 e-27$ & & & 0.002 & 0.63 & $<0.001$ & 1.05 & 0.300 & 0.62 & $<0.001$ & 0.60 & $<0.001$ & 22.71 & $<0.001$ \\
\hline WS0111_C18 & At3g17390 & $7 e-88$ & $S$-a & 1.02 & 0.884 & 1.07 & 0.652 & 1.52 & 0.017 & 0.62 & 0.009 & 0.99 & 0.924 & 2.28 & $<0.001$ \\
\hline WS0141_M06 & At5g23960 & $7 e-55$ & Terpe & 0.90 & 0.292 & 1.04 & 0.705 & 0.79 & 0.029 & 0.64 & $<0.001$ & 1.03 & 0.841 & 1.52 & 0.011 \\
\hline WS01212_L05 & Atlg72520 & $1 e-51$ & Lipox & 1. & 0.551 & 1.14 & 0.435 & 1.33 & 0.108 & 0.64 & 0.020 & 1.18 & 0.185 & 6.49 & $<0.001$ \\
\hline WS0144_K02 & At $4 \mathrm{~g}$ & & & 0. & & 0.91 & & 1.11 & 0.320 & 0.65 & 0.001 & 0.97 & 0.703 & 2.43 & $<0.001$ \\
\hline WS01116_N06 & & $1 e-110$ & & & & 1.07 & 0.549 & 0.85 & 0.188 & 0.65 & 0.003 & 0.71 & 0.009 & 2.93 & $<0.001$ \\
\hline WS0214_D12 & At2g 31100 & $2 \mathrm{e}-12$ & Putative lipase & 0.92 & 0.296 & 0.92 & 0.296 & 0.91 & 0.282 & 0.66 & $<0.001$ & 1.02 & 0.840 & 4.78 & $<0.001$ \\
\hline PX0011_E23 & At4g03260 & $4 \mathrm{e}-16$ & Leucine-rich repeat protein & 1.00 & 0.976 & 0.98 & 0.815 & 1.25 & 0.054 & 0.66 & 0.002 & 0.94 & 0.624 & 2.76 & $<0.001$ \\
\hline WS0214_C22 & At5g 40380 & $7 e-28$ & Protein kinase & 1.24 & 0.090 & 1.31 & 0.040 & 1.30 & 0.046 & 0.66 & 0.004 & 1.09 & 0.455 & 1.58 & $<0.001$ \\
\hline
\end{tabular}

${ }^{a}$ Microarray elements ranked by fold change (FC) repression by M. medusae infection at 9 dpi $(P<0.05 ; Q<0.10)$ were compared with expression profiles 2 and $24 \mathrm{~h}$ following continuous FTC feeding on poplar leaves (P. trichocarpa $\times$ P. deltoides, H11-11 genotype; S. Ralph and J. Bohlmann, unpublished). Genes in bold indicate known herbivore defense-related genes. Dark and light shading indicate significant up- and downregulation, respectively. PPO = polyphenol oxidase . dpi = days postinoculation; AGI = Arabidopsis Gene Index.

${ }^{b}$ Putative annotation of array elements assigned according to The Arabidopsis Information Resource protein set using BLASTX with a threshold of expect (E) value $<1 \mathrm{e}-05$. 
proanthocyanidins (also referred to as condensed tannins) (Winkel-Shirley 2001). To define which branch pathway is specifically upregulated in $M$. medusae-infected poplar leaves and which end products are likely to accumulate, we compre- hensively searched the 6-dpi induced-gene set for flavonoidrelated genes. Our initial annotation of array elements (Ralph et al. 2006) was manually verified with additional database searches and sequence alignments for genes involved in poplar

Table 3. Most highly induced microarray elements at each infection timepoint, ranked by fold induction ${ }^{\mathrm{a}}$

\begin{tabular}{|c|c|c|c|c|c|c|c|}
\hline Clone ID & AGI number & E-value & BLASTX vs. Arabidopsis $^{\text {b }}$ & Cluster & FC & $\boldsymbol{P}$ & $Q$ \\
\hline \multicolumn{8}{|l|}{$1 \mathrm{dpi}$} \\
\hline WS0211_H11 & n.a. & n.a. & No significant hit & 4 & 3.61 & $<0.001$ & $<0.001$ \\
\hline WS01119_K08 & At5g 10360 & $3 e-67$ & $40 \mathrm{~S}$ ribosomal protein $\mathrm{S} 6$ & 1 & 3.30 & $<0.001$ & $<0.001$ \\
\hline WS0175_A23 & At2g47170 & 1e-09 & ADP-ribosylation factor 1 & 4 & 3.19 & $<0.001$ & 0.002 \\
\hline WS0161_F14 & At3g 10740 & $3 e-19$ & Glycosyl hydrolase & 4 & 3.04 & $<0.001$ & 0.008 \\
\hline WS0153_D07 & n.a. & n.a. & No significant hit & 1 & 2.90 & $<0.001$ & 0.002 \\
\hline WS0133_I11 & At1g17860 & $3 e-06$ & Kunitz trypsin and protease inhibitor & 7 & 2.86 & $<0.001$ & 0.002 \\
\hline WS01223_D01 & Atlg74320 & $2 e-80$ & Choline kinase & 7 & 2.63 & $<0.001$ & $<0.001$ \\
\hline WS0213_B15 & n.a. & n.a. & No significant hit & 4 & 2.63 & 0.001 & 0.008 \\
\hline WS0223_H24 & n.a. & n.a. & No significant hit & 5 & 2.61 & $<0.001$ & 0.076 \\
\hline WS0222_L05 & n.a. & n.a. & No significant hit & 5 & 2.53 & $<0.001$ & 0.018 \\
\hline WS01218_H13 & At3g55990 & $7 e-09$ & Expressed protein & 2 & 2.53 & $<0.001$ & 0.017 \\
\hline WS0211_N10 & Atlg12740 & $4 e-42$ & Cytochrome P450 & 1 & 2.44 & $<0.001$ & 0.006 \\
\hline WS0154_K17 & n.a. & n.a. & No significant hit & 7 & 2.40 & $<0.001$ & 0.011 \\
\hline WS01216_O19 & n.a. & n.a. & No significant hit & 2 & 2.36 & $<0.001$ & 0.045 \\
\hline WS0163_H09 & At1g18720 & $5 e-60$ & Similar to YGL010w-like protein & 2 & 2.30 & $<0.001$ & 0.042 \\
\hline \multicolumn{8}{|l|}{3 dpi } \\
\hline WS0175_A23 & At2g47170 & 1e-09 & ADP-ribosylation factor 1 (ARF1) & 4 & 8.17 & $<0.001$ & $<0.001$ \\
\hline WS0175_O01 & At4g38620 & $1 \mathrm{e}-12$ & Myb family transcription factor (MYB4) & 8 & 4.98 & $<0.001$ & 0.002 \\
\hline WS01221_M15 & At5g24530 & $1 e-14$ & $\mathrm{~F} 3 \mathrm{H}$ & 4 & 4.93 & $<0.001$ & 0.001 \\
\hline WS0213_B15 & n.a. & n.a. & No significant hit & 4 & 4.84 & $<0.001$ & $<0.001$ \\
\hline WS0162_M02 & At5g66930 & $5 e-73$ & Expressed protein & 4 & 4.33 & $<0.001$ & 0.001 \\
\hline WS0207_E14 & n.a. & n.a. & No significant hit & 1 & 4.08 & $<0.001$ & $<0.001$ \\
\hline WS0154_N01 & At5g24530 & $4 \mathrm{e}-90$ & $\mathrm{~F} 3 \mathrm{H}$ & 4 & 4.06 & $<0.001$ & 0.025 \\
\hline WS0214_A24 & At3g56400 & $3 e-30$ & WRKY family transcription factor (WRKY4) & 2 & 3.93 & $<0.001$ & 0.038 \\
\hline WS0122_H18 & At3g57260 & $2 e-34$ & Glucan endo-1,3-beta-glucosidase, acidic isoform & 4 & 3.88 & $<0.001$ & 0.001 \\
\hline WS0162_J02 & n.a. & n.a. & No significant hit & 1 & 3.80 & 0.001 & 0.072 \\
\hline WS0208_C23 & At2g18660 & $6 e-28$ & Expansin & 4 & 3.77 & $<0.001$ & 0.016 \\
\hline WS0163_N08 & At1g36050 & $2 \mathrm{e}-52$ & Expressed protein & 4 & 3.73 & $<0.001$ & 0.001 \\
\hline WS0147_I21 & At2g14580 & $3 e-53$ & Pathogenesis-related protein 1 (PR-1) & 8 & 3.53 & $<0.001$ & $<0.001$ \\
\hline WS0145_K24 & At3g03080 & $4 e-21$ & Similar to allyl alcohol dehydrogenase & 4 & 3.35 & 0.001 & 0.080 \\
\hline WS0132_L23 & At3g57270 & $7 e-49$ & Beta-1,3-glucanase & 4 & 1.69 & $<0.001$ & $<0.001$ \\
\hline \multicolumn{8}{|l|}{6 dpi } \\
\hline WS0204_D20 & n.a. & n.a. & No significant hit & 6 & 18.70 & $<0.001$ & $<0.001$ \\
\hline WS0123_C10 & Atlg75290 & $7 e-10$ & Leucoanthocyanidin reductase & 6 & 18.58 & $<0.001$ & $<0.001$ \\
\hline WS0153_P12 & n.a. & n.a. & No significant hit & 6 & 17.88 & $<0.001$ & $<0.001$ \\
\hline WS02010_G23 & At5g13930 & $7 \mathrm{e}-08$ & Chalcone synthase / naringenin-chalcone synthase & 6 & 17.04 & $<0.001$ & $<0.001$ \\
\hline WS01127_H15 & At1g72550 & $9 \mathrm{e}-11$ & tRNA synthetase beta subunit & 6 & 16.68 & $<0.001$ & $<0.001$ \\
\hline WS0221_Fon 08 & At4g22880 & $3 e-65$ & Leucoanthocyanidin dioxygenase & 6 & 14.02 & $<0.001$ & $<0.001$ \\
\hline WS0204_I10 & n.a. & n.a. & No significant hit & 6 & 12.70 & $<0.001$ & $<0.001$ \\
\hline WS0163_E11 & At2g46150 & $9 e-14$ & Expressed protein & 6 & 11.87 & $<0.001$ & $<0.001$ \\
\hline WS0175_A21 & Atlg56200 & $1 \mathrm{e}-08$ & Expressed protein & 6 & 11.51 & $<0.001$ & $<0.001$ \\
\hline WS01212_B14 & At3g51240 & $3 e-80$ & $\mathrm{~F} 3 \mathrm{H}$ & 6 & 11.45 & $<0.001$ & $<0.001$ \\
\hline WS0205_H14 & At4g22880 & $4 e-48$ & Leucoanthocyanidin dioxygenase & 6 & 11.38 & $<0.001$ & $<0.001$ \\
\hline WS0122_P22 & n.a. & n.a. & No significant hit & 6 & 10.44 & $<0.001$ & $<0.001$ \\
\hline WS0143_M24 & At3g51240 & $2 e-36$ & $\mathrm{~F} 3 \mathrm{H}$ & 6 & 9.73 & $<0.001$ & $<0.001$ \\
\hline WS0121_D23 & At4g24220 & $5 e-48$ & Expressed protein induced upon wounding & 6 & 9.14 & $<0.001$ & $<0.001$ \\
\hline WS01212_M19 & At1g33730 & $3 e-14$ & Cytochrome P450 & 6 & 9.02 & $<0.001$ & $<0.001$ \\
\hline \multicolumn{8}{|l|}{9 dpi } \\
\hline WS0147_I21 & At2g14580 & $5 e-53$ & Pathogenesis-related protein 1 (PR-1) & 8 & 13.92 & $<0.001$ & $<0.001$ \\
\hline WS0175_O01 & At4g38620 & $1 \mathrm{e}-12$ & Myb family transcription factor (MYB4) & 8 & 11.35 & $<0.001$ & $<0.001$ \\
\hline WS0145_K24 & At3g03080 & $4 e-21$ & Allyl alcohol dehydrogenase & 4 & 9.11 & $<0.001$ & $<0.001$ \\
\hline WS0213_B15 & n.a. & n.a. & No significant hit & 4 & 8.58 & $<0.001$ & $<0.001$ \\
\hline WS01217_B16 & At5g20620 & $2 e-63$ & Polyubiquitin (UBQ4) & 8 & 8.14 & $<0.001$ & $<0.001$ \\
\hline WS0181_F07 & At4g37260 & $2 e-40$ & Myb family transcription factor (MYB73) & 8 & 8.04 & $<0.001$ & $<0.001$ \\
\hline WS0175_A23 & At2g47170 & $1 e-97$ & ADP-ribosylation factor 1 (ARF1) & 4 & 7.48 & $<0.001$ & $<0.001$ \\
\hline WS0153_P14 & At2g14610 & $1 e-56$ & Pathogenesis-related protein 1 (PR-1) & 8 & 7.24 & $<0.001$ & 0.001 \\
\hline WS0167_N13 & At3g43230 & $3 e-53$ & Zinc finger (FYVE type) & 8 & 7.00 & $<0.001$ & $<0.001$ \\
\hline WS0122_H18 & At3g57260 & $2 e-34$ & Glucan endo-1,3-beta-glucosidase, acidic isoform & 4 & 6.63 & $<0.001$ & $<0.001$ \\
\hline WS0162_J02 & n.a. & n.a. & No significant hit & 1 & 6.22 & $<0.001$ & 0.001 \\
\hline WS0119_D08 & At1g58170 & $3 e-43$ & Disease resistance-responsive / dirigent protein & 4 & 6.05 & $<0.001$ & $<0.001$ \\
\hline WS0162_K02 & At3g45600 & $6 e-45$ & Senescence-associated & 4 & 6.04 & $<0.001$ & $<0.001$ \\
\hline WS0162_M02 & At5g66930 & $5 e-73$ & Expressed protein & 4 & 6.01 & $<0.001$ & $<0.001$ \\
\hline WS0132_L23 & At3g57270 & $7 e-49$ & Beta-1,3-glucanase & 4 & 5.57 & $<0.001$ & $<0.001$ \\
\hline
\end{tabular}

${ }^{\mathrm{a}} \mathrm{F} 3 \mathrm{H}=$ naringenin 3-dioxygenase/flavonone 3-hydroxylase, dpi = days postinoculation, AGI = Arabidopsis Gene Index, FC = fold change.

${ }^{\mathrm{b}}$ Putative annotation of array elements assigned according to The Arabidopsis Information Resource protein set using BLASTX with a threshold of expect (E) value $<1 \mathrm{e}-05$. 
phenolic metabolism and was subsequently confirmed by an independent annotation of genes of phenolic metabolism in the poplar genome (Tsai et al. 2006; Tuskan et al. 2006). Based on this analysis, all enzymatic steps required for proanthocyanidin biosynthesis are represented on the Populus $15.5 \mathrm{~K}$ microarray used in this study. Furthermore, we observed upregulation for at least one gene at every enzymatic step, with the exception of cinnamate 4-hydroxylase $(\mathrm{C} 4 \mathrm{H})$ and dihydroflavonol reductase (DFR) (Fig. 4). Almost all enzymes of the flavonoid pathway are represented by more than one gene (Tsai et al. 2006). Interestingly, the most strongly $M$. medusae-induced gene family members identified here also correspond to those genes most highly induced by wounding in $P$. fremontii $\times$ angustifolia leaves (Tsai et al. 2006).

To validate our array data and to confirm the upregulation of flavonoid and phenylpropanoid pathway genes, we carried out Northern analysis on a set of biologically independent experiments using probes for selected biosynthetic genes (Fig. 5). Since the DFR genes on the array did not show any infection- induced change in expression, we included a different but previously characterized wound-induced DFR cDNA from $P$. tremuloides (PtDFR) (Peters and Constabel 2002) as a probe in the Northern analysis. Sequence comparisons indicated that this gene was present in the P. trichocarpa genome but distinct from the DFR isoforms present on the array. Relative to control leaves, all flavonoid genes tested, including PtDFR, showed higher transcript levels in infected leaves (Fig. 5). PAL, the entry point to the phenylpropanoid pathway, was also clearly induced. Thus, our Northern data confirmed the induction of flavonoid genes as observed on microarrays and also indicated that a DFR gene not present on the array was upregulated. Although we did not test additional $\mathrm{C} 4 \mathrm{H}$ probes, given that all other steps in the pathway are represented, we speculate that another $\mathrm{C} 4 \mathrm{H}$ isoform not represented on the array may be $M$. medusae-induced.

Additional validation of microarray data was performed by gene-specific quantitative real-time polymerase chain reaction (qPCR), using a set of induced defense-related and flavonoid

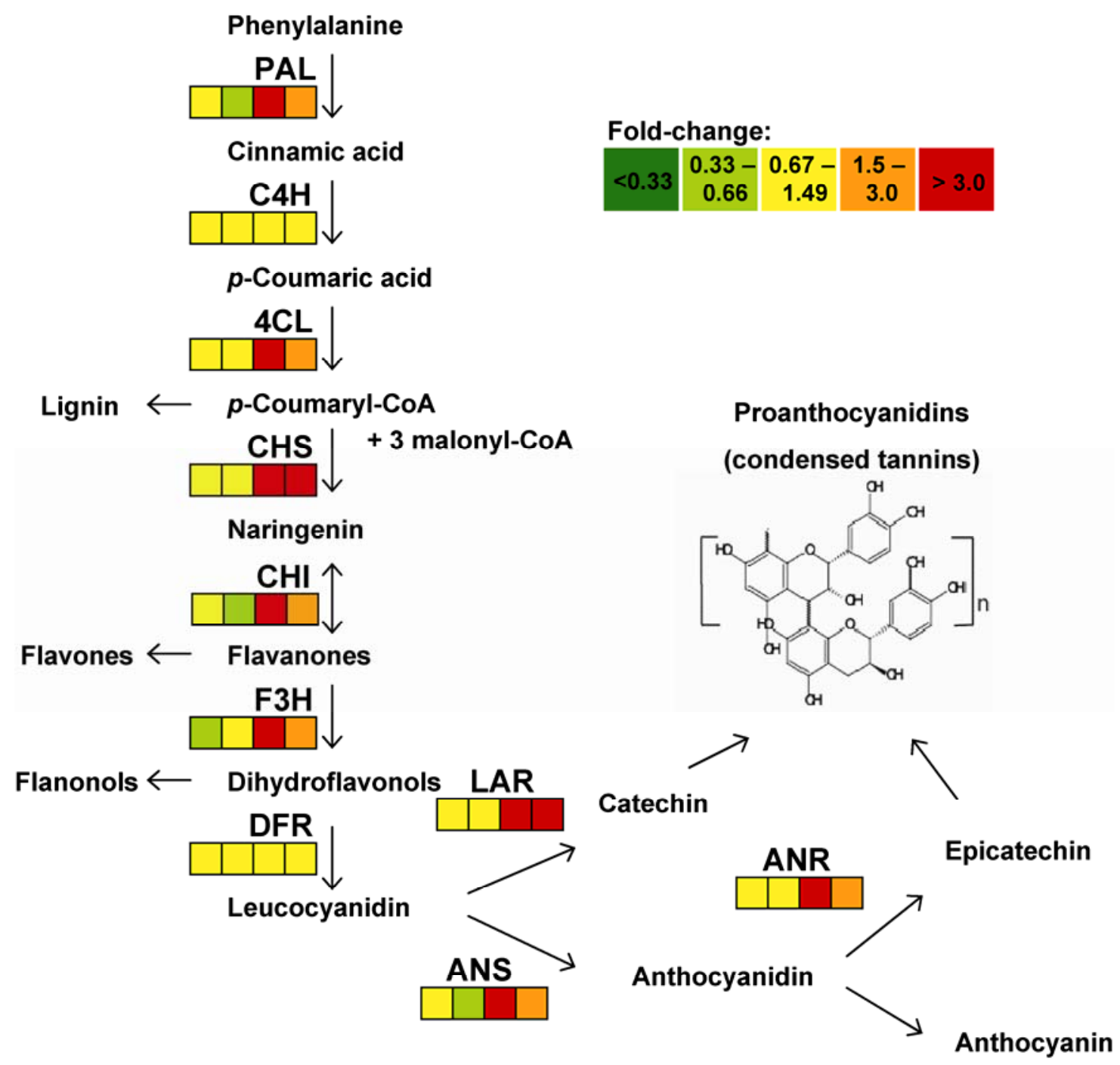

Fig. 4. Summary of expression of flavonoid biosynthetic genes in Melampsora-infected leaves of hybrid poplar. Each row of four colored boxes represents an expressed sequence tag (EST) spotted on the Populus $15.5 \mathrm{~K}$ microarray. The squares within each row represent the four timepoints (left to right, 1, 3, 6, 9 days postinoculation). A fold change color scale indicates the levels of induction (orange and red) or repression (light and dark green) in infected leaves in relation to control $(P<0.05)$. Yellow represents no significant changes. Where multiple homologs of a given gene were present on the array, the gene showing the most pronounced changes was used. $\mathrm{PAL}=$ phenylalanine ammonia-lyase (WS0154_H05), C4H = cinnamate 4-hydroxylase (WS0167_O13), 4CL = 4-coumarate-CoA ligase (WS0204_H13), CHS = chalcone synthase (WS02010_G23), CHI = chalcone-flavanone isomerase $($ WS0152_G15), F3H = flavanone 3-hydroxylase (WS01212_B14), DFR = dihydroflavonol 4-reductase (WS0175_B03), LAR = leucoanthocyanidin reductase (WS0123_C10), ANS = anthocyanidin synthase $($ WS0205_H14), and ANR = anthocyanidin reductase (WS02011_M08). 
pathway genes, as well as several noninduced genes for comparison. Although the absolute expression ratios differed somewhat between qPCR and microarray platforms, there was a good general correspondence of DE values (Table 4). The DE

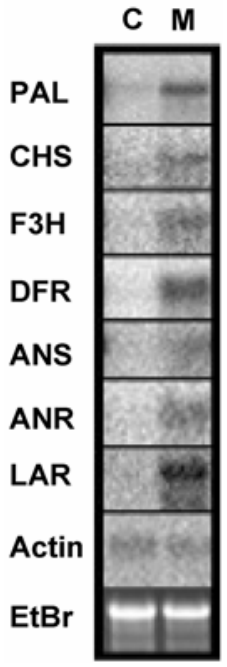

Fig. 5. Northern blot analysis demonstrating upregulation of flavonoid pathway genes by Melampsora medusae infection. RNA from leaves 9 days postinoculation was separated on agarose gels, blotted, and probed with full-length cDNAs corresponding to the indicated genes. $\mathrm{C}=$ control leaves, $\mathrm{M}=M$. medusae -infected leaves, $\mathrm{PAL}=$ phenylalanine ammonialyase (WS0154_H05), CHS = chalcone synthase (WS02010_G23), F3H = flavanone 3-hydroxylase (WS01212_B14), DFR = dihydroflavonol 4 reductase PtDFR (AY147903), LAR = leucoanthocyanidin reductase (WS0123_C10), ANS = anthocyanidin synthase (WS0205_H14), and ANR $=$ anthocyanidin reductase (WS02011_M08), Actin accession = BU $823250, \mathrm{BU} 823250, \mathrm{EtBr}=$ ethidium bromide. genes encoding PR-1, dirigent protein, F3H, glycosyl hydrolase, laccase, anthocyanidin reductase (ANR), leucoanthocyanidin reductase (LAR), and Kunitz TI showed comparable expression patterns by both techniques. For a few transcripts (e.g., PAL, Myb transcription factor, chalcone isomerase), the qPCR and microarray data disagree, in which case the induced gene expression obtained using cDNA microarrays may reflect induction of a closely related gene family member. Overall, the

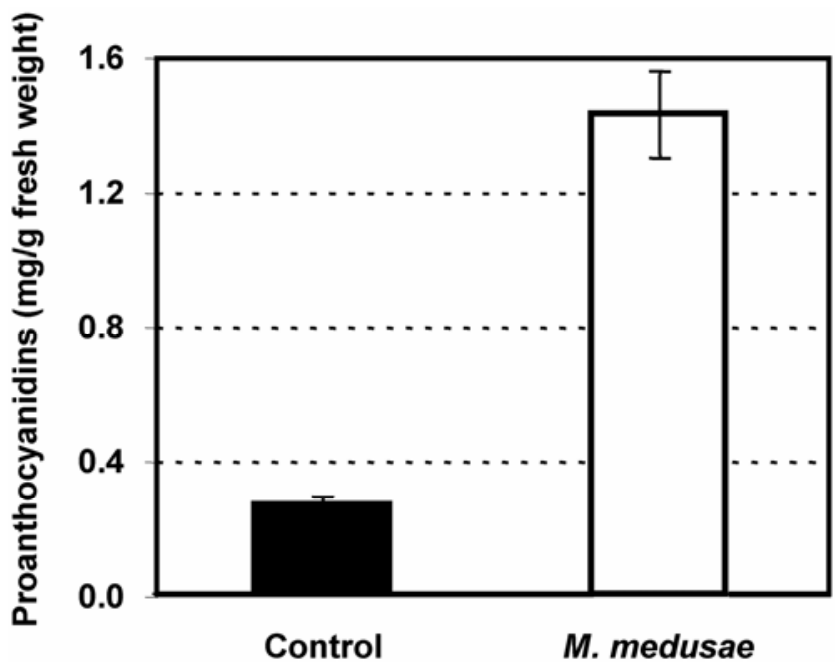

Fig. 6. Proanthocyanidin levels as determined by butanol- $\mathrm{HCl}$ assay in Melampsora medusae-infected and control poplar leaves at 20 days postinoculation. Bars show mean \pm standard error of three control or three infected leaves. This represents a 5.3-fold induction $(P<0.001)$ and is representative of multiple independent biological replicates of the experiment.

Table 4. Comparison of quantitative real-time polymerase chain reaction (PCR) and cDNA microarray analysis of gene expression in poplar leaves following infection with Melampsora medusae $e^{\mathrm{a}}$

\begin{tabular}{|c|c|c|c|c|c|c|c|c|}
\hline \multirow[b]{2}{*}{ Clone ID (annotation) } & \multicolumn{2}{|c|}{1 dpi } & \multicolumn{2}{|c|}{3 dpi } & \multicolumn{2}{|c|}{6 dpi } & \multicolumn{2}{|c|}{9 dpi } \\
\hline & FC & $\mathbf{P}$ & FC & $\mathbf{P}$ & FC & $\mathbf{P}$ & FC & $\mathbf{P}$ \\
\hline \multirow[t]{2}{*}{ WS0153_P14 (PR-1) } & 1.90 & 0.011 & 3.32 & 0.006 & 2.30 & 0.010 & 10.27 & $<0.001$ \\
\hline & 1.98 & 0.029 & 3.18 & 0.001 & 2.48 & 0.007 & 7.24 & $<0.001$ \\
\hline \multirow[t]{2}{*}{ WS0173_N04 (dirigent) } & 1.76 & 0.059 & 5.07 & 0.005 & 3.91 & 0.018 & 12.80 & $<0.001$ \\
\hline & 1.87 & 0.002 & 2.70 & $<0.001$ & 6.13 & $<0.001$ & 3.17 & $<0.001$ \\
\hline \multirow[t]{2}{*}{ WS0154_N01 (flavonone-3-hydroxylase) } & 0.77 & 0.276 & 2.64 & 0.014 & 1.21 & 0.313 & 5.15 & $<0.001$ \\
\hline & 1.50 & 0.115 & 4.06 & $<0.001$ & 2.74 & 0.001 & 3.59 & $<0.001$ \\
\hline \multirow{2}{*}{ WS0132_L23 (glycosyl hydrolase) } & 1.99 & 0.209 & 2.60 & 0.005 & 2.13 & 0.019 & 4.07 & 0.009 \\
\hline & 1.69 & $<0.001$ & 3.34 & $<0.001$ & 5.77 & $<0.001$ & 5.57 & $<0.001$ \\
\hline \multirow[t]{2}{*}{ WS01911_I09 (laccase) } & 3.70 & 0.002 & 1.54 & 0.117 & 3.97 & 0.011 & 8.23 & 0.007 \\
\hline & 1.04 & 0.821 & 1.17 & 0.392 & 1.70 & 0.011 & 2.68 & $<0.001$ \\
\hline \multirow[t]{2}{*}{ WS02011_M08 (anthocyanidin reductase) } & 0.68 & 0.112 & 0.44 & 0.005 & 5.98 & $<0.001$ & 2.16 & 0.008 \\
\hline & 0.87 & 0.723 & 0.77 & 0.527 & 5.24 & 0.002 & 1.63 & 0.247 \\
\hline \multirow[t]{2}{*}{ WS0123_C10 (leucoanthocyanidin reductase) } & 0.15 & 0.125 & 0.26 & 0.062 & 5.87 & 0.012 & 1.94 & 0.112 \\
\hline & 0.76 & 0.012 & 0.86 & 0.131 & 18.58 & $<0.001$ & 3.03 & $<0.001$ \\
\hline \multirow[t]{2}{*}{ WS0133_I11 (Kunitz protease inhibitor) } & 1.31 & 0.175 & 0.18 & 0.025 & 0.65 & 0.108 & 0.06 & 0.024 \\
\hline & 2.86 & $<0.001$ & 0.36 & $<0.001$ & 0.61 & 0.001 & 0.18 & $<0.001$ \\
\hline \multirow[t]{2}{*}{ WS0153_H17 (cinnamyl alcohol dehydrogenase) } & 0.97 & 0.410 & 1.12 & 0.250 & 0.57 & 0.045 & 1.08 & 0.331 \\
\hline & 0.98 & 0.798 & 1.00 & 0.976 & 1.38 & 0.002 & 1.42 & 0.001 \\
\hline \multirow[t]{2}{*}{ WS0155_I15 (acidic endochitinase) } & 0.75 & 0.307 & 0.53 & 0.123 & 0.30 & 0.102 & 2.18 & 0.119 \\
\hline & 1.56 & 0.174 & 1.25 & 0.484 & 1.61 & 0.144 & 0.79 & 0.453 \\
\hline \multirow{2}{*}{ WS0177_F13 (4-coumarate-CoA ligase) } & 0.73 & 0.071 & 0.59 & 0.003 & 0.64 & 0.061 & 1.39 & 0.151 \\
\hline & 1.06 & 0.711 & 1.01 & 0.952 & 1.10 & 0.561 & 1.05 & 0.748 \\
\hline \multirow[t]{2}{*}{ WS0154_H05 (phenylalanine ammonia lyase) } & 0.14 & 0.076 & 0.08 & 0.032 & 0.04 & 0.045 & 0.26 & 0.113 \\
\hline & 1.04 & 0.597 & 0.51 & $<0.001$ & 5.99 & $<0.001$ & 3.63 & $<0.001$ \\
\hline \multirow[t]{2}{*}{ WS0181_F07 (myb transcription factor) } & 0.54 & 0.091 & 0.47 & 0.060 & 2.07 & 0.023 & 0.58 & 0.007 \\
\hline & 1.97 & $<0.001$ & 3.29 & $<0.001$ & 2.74 & $<0.001$ & 8.04 & $<0.001$ \\
\hline \multirow[t]{2}{*}{ WS01210_M01 (chalcone isomerase) } & 0.11 & $<0.001$ & 0.12 & $<0.001$ & 1.15 & 0.390 & 1.00 & 0.497 \\
\hline & 1.08 & 0.792 & 0.79 & 0.416 & 4.88 & $<0.001$ & 2.01 & 0.030 \\
\hline
\end{tabular}

${ }^{a}$ Values represent fold change (FC) relative to control trees. Values obtained by real-time PCR represent the mean of three or more independent technical replicates, each consisting of pooled RNA from five biological replicates. A Student's $t$-test (two-sample, unpaired, one-sided) was performed to test significance $(P)$ of up- or downregulation of each transcript between infected and control leaves. For each transcript, real-time PCR data is shaded light gray, with the corresponding microarray data provided below. dpi = days postinoculation. 
qPCR expression profiles confirmed the trends seen on the microarray for the majority of genes. Together with the clear induction of flavonoid pathway genes demonstrated by the Northern analysis, our data suggest that the microarray data are representative of changes in gene expression caused by $M$. medusae infection.

The important observation that transcripts of the flavonoid pathway are more abundant at later timepoints following $M$. medusae infection prompted us to conduct flavonoid analysis of methanol extracts of $M$. medusae-induced and control leaves by high-pressure liquid chromatography (HPLC). Overall, the leaf phenolic profiles were very similar, and no major changes in compounds with absorption spectra corresponding to flavonoid monomers were detected (data not shown). This suggested that low-molecular-weight flavonoids do not accumulate substantially in response to the pathogen. Because the HPLC analysis employed does not readily detect larger polymeric flavonoids, we also performed butanol- $\mathrm{HCl}$ assays as an alternative means to measure proanthocyanidins (Porter et al. 1986). These assays consistently showed a strong and statistically significant increase in proanthocyanidin levels in infected leaves as compared with control leaves (Fig. 6). The induction of proanthocyanidins in response to $M$. medusae was obtained in multiple independent experiments and is consistent with induction of LAR and ANR (Figs. 4 and 5), genes specific to the proanthocyanidin pathway. Therefore, our data indicate that the induction of flavonoid pathway genes during the $M$. medusae-induced defense response leads to the accumulation of polymeric proanthocyanidins.

\section{DISCUSSION}

The recent major advances in poplar genomics have facilitated large-scale investigations of a variety of processes unique to tree biology, and recently, a few studies have addressed responses of this important tree model system to biotic stressors, such as virus infection and herbivore attack (Major and Constabel 2006; Ralph et al. 2006; Smith et al. 2004). Here, we investigate the responses of hybrid poplar to infection by the biotrophic rust fungus $M$. medusae, using a Populus $15.5 \mathrm{~K}$ gene microarray. Since similar interactions with a biotrophic fungus are not known for Arabidopsis, poplar now provides one of the best established genomic systems to study this important biological interaction. Overall, our results indicate that, while relatively few genes are DE in the first three days of rust infection, the effects on the host transcriptome were much more pronounced at 6 and 9 dpi. We determined that Melampsora infection represses many herbivore defense genes that are generally induced by wounding or insect feeding (Major and Constabel 2006; Ralph et al. 2006). Our experiments revealed that flavonoid pathway genes are strongly upregulated in the compatible poplar-M. medusae interaction, leading to an accumulation of proanthocyanidins in response to M. medusae. To the best of our knowledge, this has not previously been known to occur in the poplar pathogen-defense response.

Our histological analysis documented extensive growth of M. medusae in the leaf within the first $24 \mathrm{~h}$ (Fig. 1). Such rapid colonization of poplar leaves by a Melampsora rust fungus had also been reported by Laurans and Pilate (1999), who observed penetration of $P$. deltoides $\times P$. nigra leaf stomata by $M$. laricipopulina within $2 \mathrm{~h}$ of infection and haustoria in the leaf within $24 \mathrm{~h}$ of inoculation. We saw no visible evidence of failed defenses in the plant cells, such as partial haustorial encasements or deposition of phenolics, suggesting that the pathogen successfully avoids detection by the host or is able to suppress an early defense response. While we observed upregulation of some defense genes, such as PR-1 at 1 dpi and 3 dpi, fewer than $1 \%$ of genes were DE at either early timepoint, compared with approximately $10 \%$ DE at 6 and 9 dpi (Fig. 2). The absence of an early induced defense response was surprising, given the considerable intercellular fungal growth and penetration of host cells by haustoria by $1 \mathrm{dpi}$. This low-level response in poplar is contrasted by a much larger suite of plant genes induced within this timeframe in other compatible plantpathogen interactions. For example, with the $8 \mathrm{~K}$ Arabidopsis Affymetrix gene chip, Tao and associates (2003) identified 976 genes as induced with a virulent strain of Pseudomonas syringae within the first $30 \mathrm{~h}$ of infection. Following Arabidopsis inoculation with Alternaria brassicicola (a necrotroph), over 600 genes were found to be upregulated in the same time period (Van Wees et al. 2003). In soybean infected by Phytophthora sojae, a hemibiotrophic oomycete, almost $10 \%$ of elements on a 3,920 gene array were induced at least twofold within $24 \mathrm{~h}$ (Moy et al. 2004). In all these pathosystems, the number of genes induced within $24 \mathrm{~h}$ is significantly greater than what we observed, even when using a relatively low 1.5fold threshold. This difference was even more poignant when we reanalyzed our data using a twofold induction threshold to facilitate a direct comparison with other studies; in this analysis, only 30 elements are scored as significantly induced at 1 dpi. The low number of responding genes is not due to a weak infection, as the fungus is proliferating throughout the leaf at this time (Fig. 1) and, at the end of the experiment, the leaf is covered by pustules. Rather, this low-level response during a strong infection appears to reflect the ability of biotrophic $M$. medusae rust fungus to either actively repress or avoid stimulating a rapid and major defensive response and defenserelated gene expression in leaves of hybrid poplar.

An active role of biotrophic rust fungi in suppressing host defenses and inducing susceptibility has been established in mixed inoculation experiments in which the ability of individual cells to resist haustorial formation by an incompatible biotroph is compromised by the presence of compatible haustoria in the same or nearby cells (Heath 1983; Olesen et al. 2003). Haustoria are complex structures by which biotrophic fungi such as rusts absorb cellular nutrients from living plant cells; they also appear to mediate host defense suppression (Voegele and Mendgen 2003). The mechanism by which this occurs is not known; however, haustoria-specific proteins that appear to be transported in the plant cell have recently been identified in M. linii (Catanzariti et al. 2006; Dodds et al. 2004) and Uromyces fabae (Kemen et al. 2005). It is likely that such proteins act as pathogenicity factors and help to suppress plant defenses. Phytopathogenic bacteria can also use such factors to suppress plant defense responses, including hypersensitive cell death, papillae, and cell-wall modification (Abramovitch and Martin 2004). Although our transcript profiling data do not provide explicit clues as to a possible mechanism for the suppression of defenses, a number of kinases and other potential signaling proteins are repressed at 1 dpi relative to control leaves. Such genes provide relevant targets for future functional and mechanistic analysis of defense regulation in this system.

In contrast to surprisingly few early gene-expression changes, large changes in the host transcriptome were observed by 6 and 9 dpi (Fig. 2). An analysis of the transcriptome over the entire 9day infection timecourse indicated that $M$. medusae infection of hybrid poplar resulted in an approximately equal number of upor downregulated genes (approximately 9\%). Thus, across all timepoints, the magnitude of the transcriptional response is comparable to that observed in Arabidopsis and other species when infected with a variety of pathogens. For example, in a comprehensive study using the $8 \mathrm{~K}$ Affymetrix gene chip, Tao and associates (2003) found that approximately 2,000 genes 
were DE following inoculation with virulent, avirulent, or nonhost strains of Pseudomonas syringae. In the only other published microarray analysis of poplar-pathogen interactions, Smith and associates (2004) reported that 1,301 genes showed altered levels of expression in response to combined wounding and viral infection, while 599 responded only to viral infection. This represents 5 and $10 \%$ of the elements on a $13 \mathrm{~K}$ Populus array, which contains an estimated 9,000 to 10,000 unique genes (Andersson et al. 2004). Thus, both the present report and that of Smith and associates (2004) showed that pathogen infection of poplar leaves has a dramatic effect on gene expression, as observed in herbaceous plant-pathogen interactions.

Despite the small number of rapidly induced genes, some induced defense-related genes were evident at 3 dpi. PR-1-like genes as well as Myb and WRKY transcription factors were among the most strongly induced genes at this timepoint (Table 3). PR-1 is a common marker gene for induced plant defense against biotrophic pathogens (Van Loon et al. 2006), and both Myb and WRKY proteins are involved in activating defenses in herbaceous plants (Eulgem 2005). PR-1 genes were observed to be strongly induced throughout the infection timecourse. Analysis of the $P$. trichocarpa genome sequence (Tuskan et al. 2006) indicated that multiple PR-1-like genes are present in the genome (R. Mellway and C. P. Constabel, unpublished data). At least six of these are represented on our microarray, and four of these show induction during our timecourse experiments. Although no mechanism of action is known, studies using transgenic plants and in vitro assays with purified proteins have demonstrated an antifungal activity, including antifungal activity towards rust fungi (Rauscher et al. 1999). Other PR genes that consistently showed induced expression levels are $\beta$-1,3-glucanases, chitinases, and osmotin-like proteins; these have all been shown to possess direct antifungal activity and may act synergistically (Van Loon et al. 2006).

Whereas PR-1 and other typical pathogen defense genes were clearly upregulated at $3 \mathrm{dpi}$, we also noted the simultaneous downregulation of a suite of previously identified herbivoreinduced defense genes (Table 2). Most notably, Kunitz TI, chitinases, and PPO, all strongly herbivore-induced genes in this poplar hybrid (Christopher et al. 2004; Major and Constabel 2006; Ralph et al. 2006), are also significantly repressed by $M$. medusae at 3, 6, and 9 dpi. Poplar PPO has been demonstrated to have antiherbivore effects when overexpressed in transgenic poplar (Wang and Constabel 2004), and induced poplar Kunitz TIs are inhibitory to lepidopteran proteases (I. T. Major and C. P. Constabel, unpublished data). Likewise, the terpene synthase (-)-germacrene D synthase has been implicated in indirect defense against herbivory, based on its role in forest tent caterpillar-induced systemic volatile release (Arimura et al. 2004). Interestingly, at $1 \mathrm{dpi}$, many of these genes show significant induction by $M$. medusae, reminiscent of an early wound response that is then turned off. This pattern of initial upregulation followed by repression could also reflect an antagonism of pathogen and wound- or herbivore-induced defense responses, as observed in other plants, and which has been suggested to indicate an evolutionary trade-off between pathogen and herbivore resistance (Felton and Korth 2000). At the level of signaling, some clear antagonistic effects have been found for jasmonic acid (JA) and salicylic acid (SA), key mediators for herbivory and biotrophic pathogen defenses, respectively. In tomato, for example, SA-induced pathogen defense completely inhibited the wound induction of PI and PR protein induction was blocked by JA (Doares et al. 1995). Thaler and associates (2002) also found an inverse correlation between pest and pathogen resistance in induced plants, and in Arabidopsis, Cui and associates (2005) demonstrated greater insect susceptibility for a mutant plant expressing constitutive pathogen resistance.
The strong induction of genes encoding the flavonoid pathway enzymes and the subsequent accumulation of proanthocyanidins late in the infection was unexpected because these compounds are not typically associated with pathogen defense. Genes encoding almost the entire pathway from Phe and malonyl-CoA to proanthocyanidin synthesis were induced (Fig. 4). The last characterized enzymatic steps in proanthocyanidin synthesis are carried out by LAR and ANR, two recently identified enzymes that define parallel routes to proanthocyanidin biosynthesis (Tanner et al. 2003; Xie et al. 2003). ESTs for both enzymes are present on the array and exhibit induction following inoculation, suggesting that both enzymes participate in proanthocyanidin synthesis in poplar. This would be consistent with the structure of $P$. tremuloides proanthocyanidin, which is derived from both LAR and ANR starter subunits (Ayres et al. 1997). The induction of these proanthocyanidinspecific genes correlated well with our phytochemical analysis, which detected enhanced proanthocyanidin accumulation but no induction of other flavonoids or phenolics. The HPLC methods used are able to separate a variety of poplar phenolics and flavonoids, but HPLC profiles of control and infected leaves were virtually identical. We found no evidence for induced synthesis of lignin or monolignols, and expression of key genes of the lignin pathway such as caffeoyl-CoA $O$ methyl transferase, cinnamoyl-CoA reductase, or cinnamyl alcohol dehydrogenase typically varied by less than twofold during the infection (data not shown). A number of laccases (diphenol oxidases) were upregulated at $6 \mathrm{dpi}$ and could be involved in lignin biosynthesis or in other oxidative reactions. Overall, our gene expression and phytochemical data suggest that the induction of the flavonoid and proanthocyanidin pathway is specific and does not reflect a general stimulation of phenylpropanoid metabolism, other than the core enzymes required for flavonoid synthesis (e.g., PAL and 4CL).

A number of elements annotated as isoflavone reductase (IFR)-like genes were upregulated at 6 dpi. IFR-like proteins belong to the $\mathrm{NAD}(\mathrm{P}) \mathrm{H}$-dependant reductase/epimerase/dehydrogenase (RED) superfamily, which also includes DFR, LAR, and ANR. IFR-like genes were first annotated based on their similarity to enzymes of the isoflavonoid pathway as well as lignan reductases (Gang et al. 1999). Isoflavonoids and lignans have fairly restricted distributions in the plant kingdom and have not been reported in poplar spp., so it is likely that they are involved in other, as-yet-unknown phytochemical pathways. IFR-like genes have often been described in plants in the context of pathogen challenge and other stresses (Lers et al. 1998). Since we found no evidence of any other flavonoid accumulation in infected leaves in addition to the proanthocyanidins, induction of these genes in poplar may play a role unrelated to flavonoid metabolism.

In previous work, we documented the wound-induced synthesis of proanthocyanidins in $P$. tremuloides leaves (Peters and Constabel 2002). In that study, genes encoding enzymes of flavonoid biosynthesis were upregulated as part of the herbivory defense response, in parallel with other defense genes encoding TI and PPO. In the current study of Melampsorainfected poplar, the herbivore defense response is repressed, while the proanthocyanidin pathway is induced. This finding suggests that proanthocyanidin induction by Melampsora may not be simply a delayed wound response triggered by pathogen invasion. While proanthocyanidins are known to have general antimicrobial properties, they have not been previously reported as phytoalexins or pathogen-induced compounds. However, Hakulinen (1998) found that Melampsora infection of Salix myrsinifolia (closely related to poplars) can result in enhanced synthesis of catechin, a proanthocyanidin precursor, as well as chlorogenic acid, a nonflavonoid phenolic. In some other plant 
families, flavonoid-derived phytoalexins are prominent. In the family Fabaceae, isoflavonoid phytoalexins such as pterocarpans and isoflavones are potent fungistatic compounds (Dixon 2001). Flavonoids and related compounds have also been reported as phytoalexins in rice (Tamogami et al. 1997), sorghum (Lo et al. 1999), carnation (Curir et al. 2005), and cucumber (McNally et al. 2003); elsewhere in the plant kingdom, pathogen-induced flavonoids appear to be relatively rare. Whether proanthocyanidins contribute to Melampsora resistance in incompatible interactions and act as phytoalexins is not yet clear. The present study as well as the genetic and genomic resources now available for poplar will allow us to address this question in future works.

\section{MATERIALS AND METHODS}

\section{Plant material and rust inoculation.}

Hybrid poplar $(P$. trichocarpa [Torr. \& Gray] $\times P$. deltoides Bartr. hybrid H11-11 [University of Washington, Seattle, U.S.A.]) plants were grown in the University of Victoria's Forest Biology greenhouse under long-day conditions as described (Constabel et al. 2000). A field-collected Melampsora medusae isolate was provided by G. Newcombe, University of Idaho (Moscow, ID, U.S.A.), and was maintained on greenhouse hybrid poplar plants. Inoculation solutions were prepared by harvesting urediospores from three fully expanded leaves of a 7to 9-day-old inoculation and transferring them to $50 \mathrm{ml}$ of distilled water with a cotton swab. This solution was sprayed as a fine mist onto the abaxial surfaces of leaves of 12-week-old plants (approximately $1 \mathrm{~m}$ in height). Leaves of plastochron index 5 to 7 (Larson and Isebrands 1971) were used for experiments. Light-weight clear plastic bags were placed over the plant tops, covering the sprayed leaves, to maintain high humidity for the infection. Bags were removed $48 \mathrm{~h}$ after inoculation. Control plants were treated in the same manner but were sprayed with $50 \mathrm{ml}$ of distilled water. The entire lamina of leaves from five infected and five control plants was harvested 1, 3, 6, and $9 \mathrm{dpi}$, and the samples were flash-frozen in liquid nitrogen.

\section{Histological analyses.}

For microscopy, leaves were harvested at 1, 2, 3, 6, and 9 days postinoculation (dpi) and excised sections were placed immediately into boiling $70 \%$ ( $\mathrm{vol} / \mathrm{vol}$ ) ethanol for $5 \mathrm{~min}$. The ethanol was renewed and boiling was continued for another 5 min or until the leaf sections were colorless. Leaf sections were transferred to cold $70 \%$ alcohol for $24 \mathrm{~h}$ and then were transferred into $100 \%$ ethanol for storage. In some cases, a few drops of cotton blue in lactophenol were added to the ethanol to stain the fungus within the leaves. Leaf sections were cleared by placing them in warm, saturated aqueous chloral hydrate and leaving them in the solution for at least 2 days before mounting them on glass slides in modified Hoyer's medium (Cunningham 1972). The leaf sections were examined using a Reicher-Jung Polyvar microscope (Reicher AG, Vienna, Austria) equipped with differential interference contrast optics.

\section{RNA extraction and analysis.}

RNA extraction for microarrays and qPCR was performed following the method described by Kolosova and associates (2004). For quality control, RNA samples were labeled with ${ }^{32} \mathrm{P}$ for reverse transcription and analyzed on agarose gel electrophoresis; only samples that produced a homogeneous smear from 0.5 to $5 \mathrm{~kb}$ were used in downstream applications. For Northern analysis, RNA was extracted according to Haruta and associates (2001), except that phenol was excluded from the protocol. Northern hybridization was carried out using standard procedures and ${ }^{32} \mathrm{P}$-labeled probes (Church and Gilbert 1984; Sambrook and Russell 2001). Probes consisted of the entire coding sequences and were PCR-amplified from poplar cDNA, using primers (Supplemental Table 3) based on the $P$. trichocarpa genome sequence (Joint Genome Institute $P$. trichocarpa website), and were verified by sequence analysis.

\section{Microarray hybridization, analysis, and validation by qPCR.}

The Populus 15.5K cDNA microarray (containing 15,496 cDNA elements) and hybridization and slide scanning conditions are described in detail by Ralph and associates (2006). The cDNA libraries used to build the array came from a variety of poplar tissues and included tissues subjected to different biotic stresses, including exposure to fungal extracts. All microarray experiments were designed to comply with MIAME (minimum information about a microarray experiment) guidelines (Brazma et al. 2001). For microarray experiments, total RNA isolated from five $M$. medusae-infected and five control trees was pooled by treatment and timepoint. Four arrays, including two dye swaps, were hybridized for each timepoint, for a total of 16 hybridizations.

Before data normalization, the lowest $10 \%$ of median foreground intensities was subtracted from the median foreground intensity to correct for background intensity. Data were then normalized to compensate for nonlinearity of intensity distributions, using the variance stabilizing normalization method (Huber et al. 2002). In order to assess the biological response to $M$. medusae infection (MM), a linear model containing a dye effect and a treatment effect for MM minus control was fit for 1, 3, 6, and $9 \mathrm{dpi}$, using data from four hybridizations per timepoint. Expression variance was derived from technical variance among slides. In retrospect, it would have been preferable to use independent biological replicates throughout array analyses instead of technical replicates with pooled samples from multiple biological replicates. However, using the same Populus $15.5 \mathrm{~K}$ microarray and the same hybrid poplar genotype, we have shown that technical variation exceeds biological variation when genetically identical trees derived from clonal populations are used under greenhouse conditions in a microarray analysis of plant herbivory responses, in which nearly identical results were obtained with technical or biological replicates (Ralph et al. 2006). The ratio of each treatment parameter estimate to the standard error was used to calculate a $t$ statistic, from which a $P$ value was obtained. The $Q$ value for each effect and gene was calculated for each of the models to adjust for the false discovery rate (Storey and Tibshirani 2003). Cluster analysis was performed on a set of 2,988 array elements showing significant DE (i.e., fold change between infected and control leaves $>1.5$ and $P<0.05)$ using the divisive DIANA algorithm (Bryan 2004), and the first eight clusters that formed were examined in detail. All statistical analyses were performed within the $\mathrm{R}$ statistical package ( $\mathrm{R}$ Project website).

Validation of microarray expression data using qPCR for selected genes was performed as described previously (Ralph et al. 2006), except that primers for poplar elongation factor $1 \beta$ subunit (GenBank accession number CV270571; poplar EST WS0152_F14) were designed and served as a quantification control. Gene-specific primer sequences are provided in Supplemental Table 4.

\section{Transcript profiling \\ in poplar leaves following insect herbivory.}

To monitor gene expression in response to insect feeding, poplar plants $(P$. trichocarpa [Torr. \& Gray] $\times P$. deltoides Bartr. hybrid H11-11) were grown at the University of British 
Columbia greenhouse as described by Ralph and associates (2006) to a height of 60 to $70 \mathrm{~cm}$. Forest tent caterpillars (FTC; Malacosoma disstria Hübner) were obtained from the Great Lakes Forestry Center (NRCan, Sault Ste. Marie, Canada) and were maintained as described by Ralph and associates (2006). For FTC treatment, groups of third to fifth instar larvae were kept without food on moist filter paper for $40 \mathrm{~h}$ prior to placing them on trees. Five FTC were added to each of five trees per timepoint (i.e., 2, 6, and $24 \mathrm{~h}$ ) under mesh bags on individual trees, with an additional set of five trees per timepoint also covered with mesh bags but otherwise left untreated as a control. Cages were placed on the five lowest, fully expanded, healthy leaves of each tree. The entire lamina of leaves were individually harvested from each tree, separately flash-frozen in liquid nitrogen, and were stored at $-80^{\circ} \mathrm{C}$ prior to RNA isolation following the protocol of Kolosova and associates (2004). For each treatment and timepoint, equal amounts of total RNA were combined from each tree prior to cDNA microarray analysis.

Hybridizations using the Populus $15.5 \mathrm{~K}$ array were performed by comparing total RNA from untreated control leaves and FTC-treated leaves for each timepoint, using four technical replicate hybridizations for each comparison with dye flips (12 slides total). Total RNA from FTC-treated leaves was also compared across the three timepoints, using two technical replicate hybridizations for each comparison with dye flips (six slides total). Hybridization conditions and slide scanning were performed as described previously (Ralph et al. 2006), and signal intensity correction and data normalization were performed as described above for Melampsora-infected leaves. To assess the biological response to FTC feeding, a linear model containing a dye effect and treatment effect for FTC minus control was fit as described above for Melampsora-infected leaves using the $\mathrm{R}$ statistical package. A complete description of the genomic analysis of FTC herbivory in poplar will be described elsewhere (S. Ralph and J. Bohlmann, unpublished data).

\section{Phytochemical analysis.}

For phytochemical assays, frozen leaf tissue was ground in liquid nitrogen prior to extraction with $80 \%$ methanol for $2 \mathrm{~h}$. Extracts were centrifuged to remove solid debris, and proanthocyanidins were assayed using the butanol- $\mathrm{HCl}$ assay as described (Peters and Constabel 2002; Porter et al. 1986), using purified $P$. tremuloides proanthocyanidin as a standard.

\section{ACKNOWLEDGMENTS}

The authors thank G. Newcombe, University of Idaho, for advice and providing the M. medusae isolate, L. Tran and B. Binges for their help in maintaining greenhouse material, $\mathrm{H}$. Yueh for technical support, D. Aeschliman for statistical assistance, and S. Jancsik for assistance with manuscript preparation. We gratefully acknowledge the Natural Sciences and Engineering Research Council (NSERC) of Canada for funding in the form of a Genomics Project grant (to C. P. Constabel) and Discovery grants (to C. P. Constabel and J. Bohlmann), and Genome Canada and Genome British Columbia for support of the Treenomix and Treenomix Conifer Forest Health Projects (to J. Bohlmann). J. Bohlmann is an NSERC Steacie-Memorial Fellow.

\section{LITERATURE CITED}

Abramovitch, R. B., and Martin, G. B. 2004. Strategies used by bacterial pathogens to suppress plant defenses. Curr. Opin. Plant Biol. 7:356-364.

Andersson, A., Keskitalo, J., Sjödin, A., Bhalerao, R., Sterky, F., Wissel, K., Tandre, K., Aspeborg, H., Moyle, R., Ohmiya, Y., Bhalerao, R., Brunner, A., Gustafsson, P., Karlsson, J., Lundeberg, J., Nilsson, O., Sandberg, G., Strauss, S., Sundberg, B., Uhlen, M., Jansson, S., and Nilsson, P. 2004. A transcriptional timetable of autumn senescence. Genome Biol. 5:R24.

Andersson-Gunnerås, S., Mellerowicz, E. J., Love, J., Segerman, B.,
Ohmiya, Y., Coutinho, P. M., Nilsson, P., Henrissat, B., Moritz, T., and Sundberg, B. 2006. Biosynthesis of cellulose-enriched tension wood in Populus: Global analysis of transcripts and metabolites identifies biochemical and developmental regulators in secondary wall biosynthesis. Plant J. 45:144-165.

Arimura, G., Huber, D. P. W., and Bohlmann, J. 2004. Forest tent caterpillars (Malacosoma disstria) induce local and systemic diurnal emissions of terpenoid volatiles in hybrid poplar (Populus trichocarpa $\times$ deltoides): cDNA cloning, functional characterization, and patterns of gene expression of (-)-germacrene D synthase, PtdTPS1. Plant J. 37:603616.

Ayres, M. P., Clausen, T. P., MacLean, S. F., Redman, A. M., and Reichardt, P. B. 1997. Diversity of structure and antiherbivore activity in condensed tannins. Ecology 78:1696-1712.

Brazma, A., Hingamp, P., Quackenbush, J., Sherlock, G., Spellman, P., Stoeckert, C., Aach, J., Ansorge, W., Ball, C. A., Causton, H. C., Gaasterland, T., Glenisson, P., Holstege, F. C. P., Kim, I. F., Markowitz, V., Matese, J. C., Parkinson, H., Robinson, A., Sarkans, U., SchulzeKremer, S., Stewart, J., Taylor, R., Vilo, J., and Vingron, M. 2001. Minimum information about a microarray experiment (MIAME) - Towards standards for microarray data. Nat. Genet. 29:365-371.

Brosché, M., Vinocur, B., Alatalo, E. R., Lamminmäki, A., Teichmann, T., Ottow, E. A., Djilianov, D., Afif, D., Bogeat-Triboulot, M. B., Altman, A., Polle, A., Dreyer, E., Rudd, S., Paulin, L., Auvinen, P., and Kangasjärvi, J. 2005. Gene expression and metabolite profiling of Populus euphratica growing in the Negev desert. Genome Biol. 6:R101.

Brunner, A. M., Busov, V. B., and Strauss, S. H. 2004. Poplar genome sequence: Functional genomics in an ecologically dominant plant species. Trends Plant Sci. 9:49-56.

Bryan, J. 2004. Problems in gene clustering based on gene expression data. J. Multivar. Anal. 90:44-66.

Busov, V. B., Brunner, A. M., Meilan, R., Filichkin, S., Ganio, L., Gandhi, S., and Strauss, S. H. 2005. Genetic transformation: A powerful tool for dissection of adaptive traits in trees. New Phytol. 167:9-18.

Callan, B. E. 1998. Page 157 in: Diseases of Populus in British Columbia: A Diagnostic Manual. Natural Resources Canada, Canadian Forest Service, Pacific Forestry Centre, Victoria, BC, Canada.

Catanzariti, A. M., Dodds, P. N., Lawrence, G. J., Ayliffe, M. A., and Ellis, J. G. 2006. Haustorially expressed secreted proteins from flax rust are highly enriched for avirulence elicitors. Plant Cell 18:243-256.

Chisholm, S. T., Coaker, G., Day, B., and Staskawicz, B. J. 2006. Host-microbe interactions: Shaping the evolution of the plant immune response. Cell 124:803-814.

Christopher, M. E., Miranda, M., Major, I. T., and Constabel, C. P. 2004. Gene expression profiling of systemically wound-induced defenses in hybrid poplar. Planta 219:936-947.

Church, G. M., and Gilbert, W. 1984. Genomic sequencing. Proc. Natl. Acad. Sci. U.S.A. 81:1991-1995.

Constabel, C. P., Yip, L., Patton, J. J., and Christopher, M. E. 2000. Polyphenol oxidase from hybrid poplar. Cloning and expression in response to wounding and herbivory. Plant Physiol. 124:285-295.

Cui, J., Bahrami, A. K., Pringle, E. G., Hernandez-Guzman, G., Bender, C. L., Pierce, N. E., and Ausubel, F. M. 2005. Pseudomonas syringae manipulates systemic plant defenses against pathogens and herbivores. Proc. Natl. Acad. Sci. U.S.A. 102:1791-1796.

Cunningham, J. L. 1972. Miracle mounting fluid for permanent wholemounts of microfungi. Mycologia 64:906-911.

Curir, P., Dolci, M., and Galeotti, F. 2005. A phytoalexin-like flavonol involved in the carnation (Dianthus caryophyllus)-Fusarium oxysporum $\mathrm{f}$. sp. dianthi pathosystem. J. Phytopathol. 153:65-67.

Dixon, R. A. 2001. Natural products and plant disease resistance. Nature 411:843-847.

Doares, S. H., Narvaez-Vasquez, J., Conconi, A., and Ryan, C. A. 1995. Salicylic-acid inhibits synthesis of proteinase-inhibitors in tomato leaves induced by systemin and jasmonic acid. Plant Physiol. 108:17411746.

Dodds, P. N., Lawrence, G. J., Catanzariti, A. M., Ayliffe, M. A., and Ellis, J. G. 2004. The Melampsora lini AvrL567 avirulence genes are expressed in haustoria and their products are recognized inside plant cells. Plant Cell 16:755-768.

Eulgem, T. 2005. Regulation of the Arabidopsis defense transcriptome. Trends Plant Sci. 10:71-78.

Felton, G. W., and Korth, K. L. 2000. Trade-offs between pathogen and herbivore resistance. Curr. Opin. Plant Biol. 3:309-314.

Frey, P., Gérard, P., Feau, N., Husson, C., and Pinon, J. 2005. Variability and population biology of Melampsora rusts on poplars. Pages 63-72 in: Rust Diseases of Willow and Poplar. M. H. Pei and A. R. McCracken, eds. CABI Publishing, Cambridge, MA, U.S.A.

Gang, D. R., Kasahara, H., Xia, Z. Q., Vander Mijnsbrugge, K., Bauw, G., Boerjan, W., Van Montagu, M., Davin, L. B., and Lewis, N. G. 1999. 
Evolution of plant defense mechanisms-relationships of phenylcoumaran benzylic ether reductases to pinoresinol-lariciresinol and isoflavone reductases. J. Biol. Chem. 274:7516-7527.

Gilchrist, E. J., Haughn, G. W., Ying, C. C., Otto, S. P., Zhuang, J., Cheung, D., Hamberger, B., Aboutorabi, F., Kalynyak, T., Johnson, L., Bohlmann, J., Ellis, B. E., Douglas, C. J., and Cronk, Q. C. B. 2006. Use of ecotilling as an efficient SNP discovery tool to survey genetic variation in wild populations of Populus trichocarpa. Mol. Ecol. 15:1365-1376.

Glazebrook, J., Chen, W., Estes, B., Chang, H. S., Nawrath, C., Métraux, J. P., Zhu, T., and Katagiri, F. 2003. Topology of the network integrating salicylate and jasmonate signal transduction derived from global expression phenotyping. Plant J. 34:217-228.

Gu, R., Fonseca, S., Puskás, L. G., Hackler, L., Zvara, A., Dudits, D., and Pais, M. S. 2004. Transcript identification and profiling during salt stress and recovery of Populus euphratica. Tree Physiol. 24:265-276.

Hakulinen, J. 1998. Nitrogen-induced reduction in leaf phenolic level is not accompanied by increased rust frequency in a compatible willow (Salix myrsinifolia)-Melampsora rust interaction. Physiol. Plant. 102:101-110

Han, K. H., Meilan, R., Ma, C., and Strauss, S. H. 2000. An Agrobacterium tumefaciens transformation protocol effective on a variety of cottonwood hybrids (genus Populus). Plant Cell Rep. 19:315-320.

Haruta, M., Major, I. T., Christopher, M. E., Patton, J. J., and Constabel, C. P. 2001. A Kunitz trypsin inhibitor gene family from trembling aspen (Populus tremuloides Michx.): Cloning, functional expression, and induction by wounding and herbivory. Plant Mol. Biol. 46:347-359.

Heath, M. C. 1983. Relationship between developmental stage of the bean rust fungus and increased susceptibility of surrounding bean tissue to the cowpea rust fungus. Physiol. Plant Pathol. 22:45-50.

Heath, M. C. 2000. Nonhost resistance and nonspecific plant defenses. Curr. Opin. Plant Biol. 3:315-319.

Hertzberg, M., Aspeborg, H., Schrader, J., Andersson, A., Erlandsson, R., Blomqvist, K., Bhalerao, R., Uhlén, M., Teeri, T. T., Lundeberg, J., Sundberg, B., Nilsson, P., and Sandberg, G. 2001. A transcriptional roadmap to wood formation. Proc. Natl. Acad. Sci. U.S.A. 98:1473214737.

Huber, W., von Heydebreck, A., Sultmann, H., Poustka, A., and Vingron, M. 2002. Variance stabilization applied to microarray data calibration and to the quantification of differential expression. Bioinformatics 18:S96-S104.

Katagiri, F. 2004. A global view of defense gene expression regulation-A highly interconnected signaling network. Curr. Opin. Plant Biol. 7:506511

Kemen, E., Kemen, A.C., Rafiqi, M., Hempel, U., Mendgen, K., Hahn, M., and Voegele, R. T. 2005. Identification of a protein from rust fungi transferred from haustoria into infected plant cells. Mol. Plant-Microbe Interact. 18:1130-1139.

Kohler, A., Delaruelle, C., Martin, D., Encelot, N., and Martin, F. 2003 The poplar root transcriptome: Analysis of 7000 expressed sequence tags. FEBS (Fed. Eur. Biochem. Soc.) Lett. 542:37-41.

Kolosova, N., Miller, B., Ralph, S., Ellis, B. E., Douglas, C., Ritland, K., and Bohlmann, J., 2004. Isolation of high-quality RNA from gymnosperm and angiosperm trees. Biotechniques 36:821-824

Larson, P. I., and Isebrands, J. G. 1971. The plastochron index as applied to developmental studies of cottonwood. Can. J. For. Res. 1:1-11.

Laurans, E., and Pilate, G. 1999. Histological aspects of a hypersensitive response in poplar to Melampsora larici-populina. Phytopathology 89:233-238

Lers, A., Burd, S., Lomaniec, E., Droby, S., and Chalutz, E. 1998. The expression of a grapefruit gene encoding an isoflavone reductase-like protein is induced in response to UV irradiation. Plant Mol. Biol. 36:847856

Lo, S. C. C., De Verdier, K., and Nicholson, R. L. 1999. Accumulation of 3-deoxyanthocyanidin phytoalexins and resistance to Colletotrichum sublineolum in sorghum. Physiol. Mol. Plant Pathol. 55:263-273.

Major, I. T., and Constabel, C. P. 2006. Molecular analysis of poplar defense against herbivory. Comparison of wound- and insect elicitorinduced gene expression. New Phytol. 172:617-635.

Maleck, K., Levine, A., Eulgem, T., Morgan, A., Schmid, J., Lawton, K A., Dangl, J. L., and Dietrich, R. A. 2000. The transcriptome of Arabidopsis thaliana during systemic acquired resistance. Nature Genet. 26:403-410.

McNally, D. J., Wurms, K. V., Labbé, C., and Bélanger, R. R. 2003. Synthesis of $C$-glycosyl flavonoid phytoalexins as a site-specific response to fungal penetration in cucumber. Physiol. Mol. Plant Pathol. 63:293303

Mellersh, D. G., and Heath, M. C. 2003. An investigation into the involvement of defense signaling pathways in components of the nonhost resistance of Arabidopsis thaliana to rust fungi also reveals a model system for studying rust fungal compatibility. Mol. Plant-Microbe Interact. 16:398-404.

Moy, P., Qutob, D., Chapman, B. P., Atkinson, I., and Gijzen, M. 2004 Patterns of gene expression upon infection of soybean plants by Phytophthora sojae. Mol. Plant-Microbe Interact. 17:1051-1062.

Newcombe, G. 1996. The specificity of fungal pathogens of Populus. Pages 223-246 in: Biology of Populus. R. Stettler, H. D. Bradshaw, P. E. Heilman, and T. M. Hinkley, eds. National Research Council Press, Ottawa, Canada

Newcombe, G., Chastagner, G. A., Schuette, W., and Stanton, B. J. 1994 Mortality among hybrid poplar clones in a stool bed following leaf rust caused by Melampsora medusae f sp. deltoidae. Can. J. For. Res. 24:1984-1987.

Newcombe, G., Bradshaw, H. D., Chastagner, G. A., and Stettler, R. F 1996. A major gene for resistance to Melampsora medusae f. sp. deltoidae in a hybrid poplar pedigree. Phytopathology 86:87-94.

Nimchuk, Z., Eulgem, T., Holt, B. F., and Dang1, J. L. 2003. Recognition and response in the plant immune system. Annu. Rev. Genet. 37:579609.

O'Connell, R. J., and Panstruga, R. 2006. Tête a tête inside a plant cell: Establishing compatibility between plants and biotrophic fungi and oomycetes. New Phytol. 171:699-718.

Olesen, K. L., Carver, T. L. W., and Lyngkjaer, M. F. 2003. Fungal suppression of resistance against inappropriate Blumeria graminis formae speciales in barley, oat and wheat. Physiol. Mol. Plant Pathol. 62:37-50.

Peters, D. J., and Constabel, C. P. 2002. Molecular analysis of herbivoreinduced condensed tannin synthesis: Cloning and expression of dihydroflavonol reductase from trembling aspen (Populus tremuloides). Plant J. 32:701-712.

Porter, L. J., Hrstich, L. N., and Chan, B. G. 1986. The conversion of procyanidins and prodelphinidins to cyanidin and delphinidin. Phytochemistry 25:223-230.

Ralph, S., Oddy, C., Cooper, D., Yueh, H., Jancsik, S., Kolosova, N., Philippe, R. N., Aeschliman, D., White, R., Huber, D., Ritland, C. E. Benoit, F., Rigby, T., Nantel, A., Butterfield, Y. S. N., Kirkpatrick, R. Chun, E., Liu, J., Palmquist, D., Wynhoven, B., Stott, J., Yang, G., Barber, S., Holt, R. A., Siddiqui, A., Jones, S. J. M., Marra, M. A., Ellis, B. E., Douglas, C. J., Ritland, K., and Bohlmann, J. 2006. Genomics of hybrid poplar (Populus trichocarpa $\times$ deltoides) interacting with forest tent caterpillars (Malacosoma disstria): Normalized and full-length cDNA libraries, expressed sequence tags, and a cDNA microarray for the study of insect-induced defences in poplar. Mol. Ecol. 15:12751297.

Rauscher, M., Ádám, A. L., Wirtz, S., Guggenheim, R., Mendgen, K., and Deising, H. B. 1999. PR-1 protein inhibits the differentiation of rust infection hyphae in leaves of acquired resistant broad bean. Plant J. 19:625-633.

Restrepo, S., Myers, K. L., del Pozo, O., Martin, G. B., Hart, A. L., Buell, C. R., Fry, W. E., and Smart, C. D. 2005. Gene profiling of a compatible interaction between Phytophthora infestans and Solanum tuberosum suggests a role for carbonic anhydrase. Mol. Plant-Microbe Interact. 18:913-922.

Sambrook, J., and Russell, D. W. 2001. Molecular Cloning: A Laboratory Manual, 3rd ed. Cold Spring Harbor Press, Cold Spring Harbor, NY, U.S.A.

Schenk, P. M., Kazan, K., Wilson, I., Anderson, J. P., Richmond, T., Somerville, S. C., and Manners, J. M. 2000. Coordinated plant defense responses in Arabidopsis revealed by microarray analysis. Proc. Natl. Acad. Sci. U.S.A. 97:11655-11660.

Schenk, P. M., Kazan, K., Manners, J. M., Anderson, J. P., Simpson, R. S., Wilson, I. W., Somerville, S. C., and Maclean, D. J. 2003. Systemic gene expression in Arabidopsis during an incompatible interaction with Alternaria brassicicola. Plant Physiol. 132:999-1010.

Smith, C. M., Rodriguez-Buey, M., Karlsson, J., and Campbell, M. M. 2004. The response of the poplar transcriptome to wounding and subsequent infection by a viral pathogen. New Phytol. 164:123-136.

Sterky, F., Bhalerao, R. R., Unneberg, P., Segerman, B., Nilsson, P., Brunner, A. M., Charbonnel-Campaa, L., Lindvall, J. J., Tandre, K., Strauss, S. H., Sundberg, B., Gustafsson, P., Uhlén, M., Bhalerao, R. P., Nilsson, O., Sandberg, G., Karlsson, J., Lundeberg, J., and Jansson, S. 2004. A Populus EST resource for plant functional genomics. Proc. Natl. Acad. Sci. U.S.A. 101:13951-13956.

Storey, J. D., and Tibshirani, R. 2003. Statistical significance for genomewide studies. Proc. Natl. Acad. Sci. U.S.A. 99:11640-11645

Strauss, S. H., and Martin, F. M. 2004. Poplar genomics comes of age. New Phytol. 164:1-4

Tamogami, S., Rakwal, R., and Kodama, O. 1997. Phytoalexin production elicited by exogenously applied jasmonic acid in rice leaves (Oryza sativa) is under the control of cytokinins and ascorbic acid. FEBS (Fed. Eur. Biochem. Soc.) Lett. 412:61-64. 
Tanner, G. J., Francki, K. T., Abrahams, S., Watson, J. M., Larkin, P. J., and Ashton, A. R. 2003. Proanthocyanidin biosynthesis in plants-Purification of legume leucoanthocyanidin reductase and molecular cloning of its cDNA. J. Biol. Chem. 278:31647-31656.

Tao, Y., Xie, Z. Y., Chen, W., Glazebrook, J., Chang, H. S., Han, B., Zhu, T., Zou, G., and Katagiri, F. 2003. Quantitative nature of Arabidopsis responses during compatible and incompatible interactions with the bacterial pathogen Pseudomonas syringae. Plant Cell 15:317-330.

Thaler, J. S., Fidantsef, A. L., and Bostock, R. M. 2002. Antagonism between jasmonate- and salicylate-mediated induced plant resistance: Effects of concentration and timing of elicitation on defense-related proteins, herbivore, and pathogen performance in tomato. J. Chem. Ecol. 28:1131-1159.

Thilmony, R., Underwood, W., and He, S. Y. 2006. Genome-wide transcriptional analysis of the Arabidopsis thaliana interaction with the plant pathogen Pseudomonas syringae pv. tomato DC3000 and the human pathogen Escherichia coli O157:H7. Plant J. 46:34-53.

Tsai, C. J., Harding, S. A., Tschaplinski, T. J., Lindroth, R. L., and Yuan, Y. 2006. Genome-wide analysis of the structural genes regulating defense phenylpropanoid metabolism in Populus. New Phytol. 172:47-62.

Tuskan, G. A., DiFazio, S. P., and Teichmann, T. 2004. Poplar genomics is getting popular: The impact of the poplar genome project on tree research. Plant Biol. 6:2-4.

Tuskan, G. A., DiFazio, S., Jansson, S., Bohlmann, J., Grigoriev, I., Hellsten, U., Putnam, N., Ralph, S., Rombauts, S., Salamov, A., Schein, J., Sterck, L., Aerts, A., Bhalerao, R. R., Bhalerao, R. P., Blaudez, D., Boerjan, W., Brun, A., Brunner, A., Busov, V., Campbell, M., Carlson, J., Chalot, M., Chapman, J., Chen, et al. 2006. The genome of black cottonwood, Populus trichocarpa (Torr. \& Gray). Science, 313:15961604.
Van Loon, L. C., Rep, M., and Pieterse, C. M. J. 2006. Significance of inducible defense-related proteins in infected plants. Annu. Rev. Phytopathol. 44:135-162.

Van Wees, S. C. M., Chang, H. S., Zhu, T., and Glazebrook, J. 2003. Characterization of the early response of Arabidopsis to Alternaria brassici cola infection using expression profiling. Plant Physiol. 132:606-617.

Voegele, R. T., and Mendgen, K. 2003 Rust haustoria: Nutrient uptake and beyond. New Phytol. 159:93-100.

Wang, J., and Constabel, C. P. 2004. Polyphenol oxidase overexpression in transgenic Populus enhances resistance to herbivory by forest tent caterpillar (Malacosoma disstria). Planta 220:87-96.

Winkel-Shirley, B. 2001. Flavonoid biosynthesis. A colorful model for genetics, biochemistry, cell biology, and biotechnology. Plant Physiol. 126:485-493

Xie, D. Y., Sharma, S. B., Paiva, N. L., Ferreira, D., and Dixon, R. A 2003. Role of anthocyanidin reductase, encoded by BANYULS in plant flavonoid biosynthesis. Science 299:396-399.

Yin, T. M., DiFazio, S. P., Gunter, L. E., Jawdy, S. S., Boerjan, W., and Tuskan, G. A. 2004. Genetic and physical mapping of Melampsora rust resistance genes in Populus and characterization of linkage disequilibrium and flanking genomic sequence. New Phytol. 164:95-105.

\section{AUTHOR-RECOMMENDED INTERNET RESOURCES}

R Project for Statistical Computing website: www.r-project.org The Arabidopsis Information Resource (TAIR) website; www.arabidopsis.org

U.S. Department of Energy Joint Genome Institute $P$. trichocarpa webpage: www.jgi.doe.gov/poplar 